\title{
Lanthanide surrogate development for actinide aerosol waste
}

\author{
Timothy J. Boyle, Josh Hubbard, \\ Xavier J. Robinson, Fernando (NMI) Guerrero, Taylor Settecerri
}

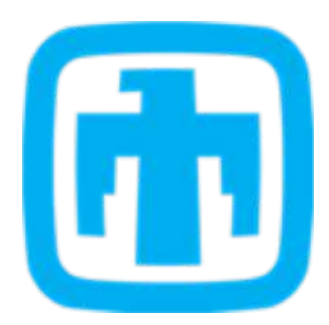

SAND2019-12221 A

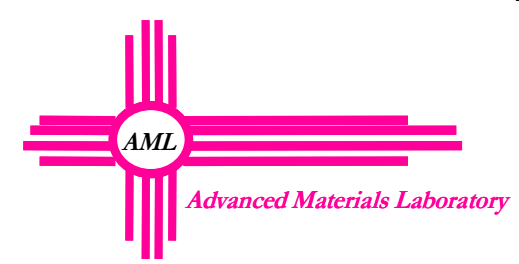

\section{Sandia National Laboratories}

Advanced Materials Laboratory

1001 University Blvd SE

Albuquerque, NM 87106

tjboyle@sandia.gov

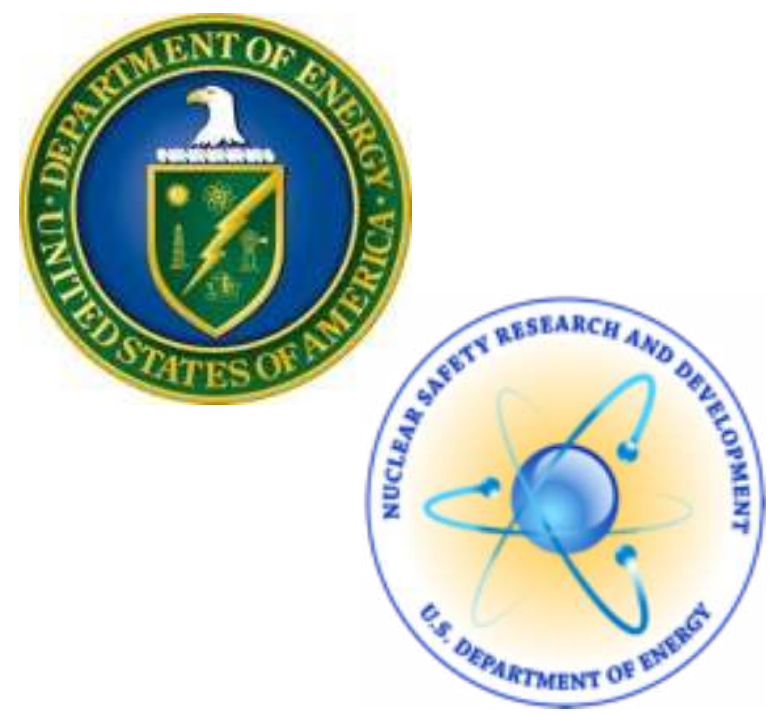

This work was funded by the Nuclear Safety Research and Development (NSR\&D) Program which is managed by the Office of Nuclear Safety, within the Office of

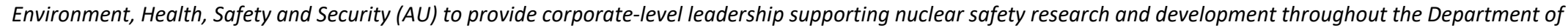
Energy (DOE). Sandia National Laboratories is a multi-mission laboratory managed and operated by National Technology and Engineering Solutions of Sandia, LLC., a

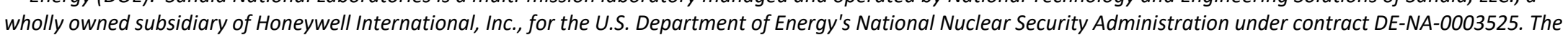
views expressed in the presentation do not necessarily represent the views of the U.S. Department of Energy or the United States Government 


\section{"Red Oil" explosions have occurred in liquid-liquid extraction of actinide wastes}

\section{$\mathrm{UO}_{2}\left(\mathrm{NO}_{3}\right)_{2}+\mathrm{TBP} /$ kerosene $\longrightarrow$}

- red oil: a substance of nonspecific composition formed when an organic phase consisting of TBP and diluent is in contact with concentrated nitric acid is heated above $120^{\circ} \mathrm{C}$ under reflux.

- The red color imparted to the organic phase is believed to be nitrated organic species.

- produced in contact with less than $10 \mathrm{M}$ nitric acid, but only at temperatures above $137^{\circ} \mathrm{C}($ Enos, 2002)

- Red oil can also be produced with pure TBP in contact with boiling 14.9 $\mathrm{M}$ nitric acid under total reflux.
Six explosions have occurred world wide:

- Hanford Site (1953)

- Savannah River Site (1953 and 1975)

- Tomsk-7 Seversk Russia (1993)

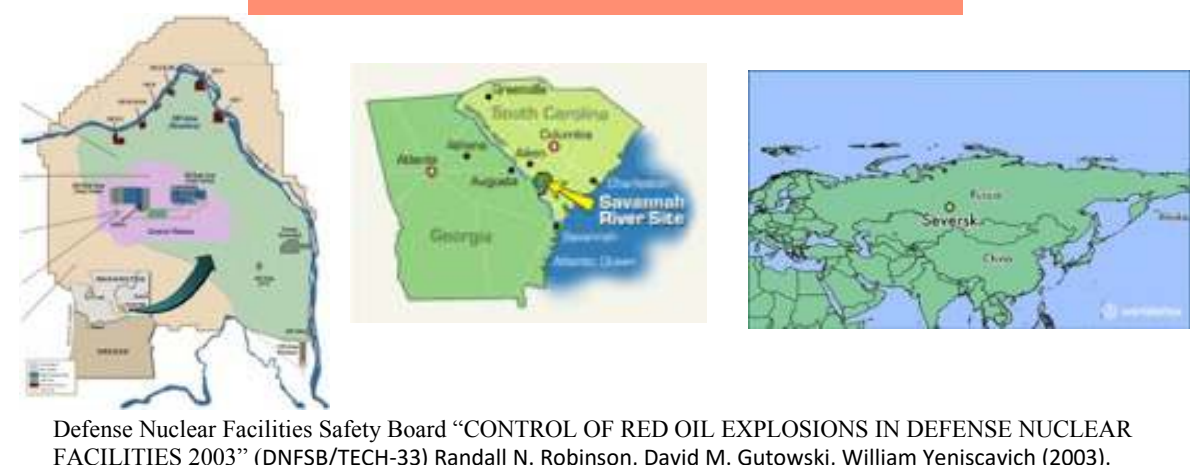

Conditions for Runaway Red Oil Explosion

- The presence of TBP in organic phase

- Organic phase in contact with nitric acid greater than $10 \mathrm{M}$

- Solution temperature greater than $130^{\circ} \mathrm{C}$

- Insufficient venting area

\section{Exacerbators}

- The presence of a diluent

- The presence of metal ions in the aqueous phase that can solvate with TBP in the organic phase and cause phase inversion

\section{Controls}

- Temperature: maintain at less than $130^{\circ} \mathrm{C}$.

- Pressure: provide a sufficient vent for the process.

- Mass: remove organics from the process.

- Concentration: maintain nitric acid less than $10 \mathrm{M}$. 


\section{BNWL-B-274 report from 1973 is the basis of actinide aerosol particulate waste release data.}

BNWL-B-274 describes the original experimental apparatus (1973). Shown below.

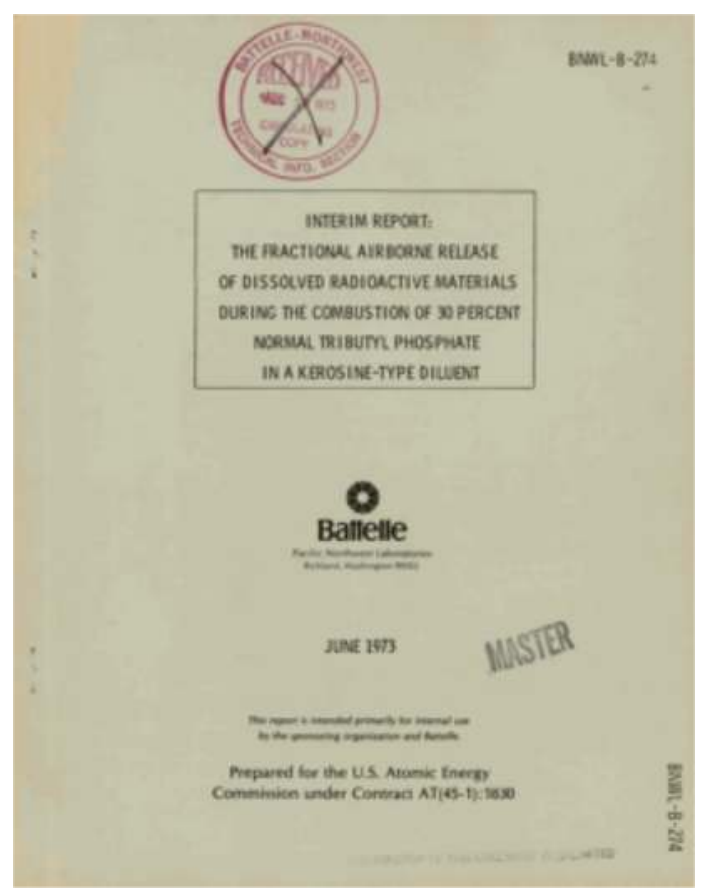

- The fractional airborne release of the isotopes evaluated was $<1 \%$ except for ${ }^{131}$ ( (lost up to the time of self-extinguishment ( $\left.65 \%\right)$ ).

- lodine sublimes at $84^{\circ} \mathrm{C}$ and methyl iodide boils at $42.5^{\circ} \mathrm{C}$, high releases are not surprising.

- The fractional release of the remaining materials - $U, C s, C e, Z r$ - were low.

- Ce appears to be the most readily entrained but considerable variations can be found in the measured release for a II materials.

- $U$ tends to remain with the $n$-TBP which is less mobile than the kerosine.

- The fractional release of $U$ is an order of magnitude less up to the point of self-extinguishment than for the other elements.

- Up to the point of extinguishment the TBP appears to be little involved in the burning process.

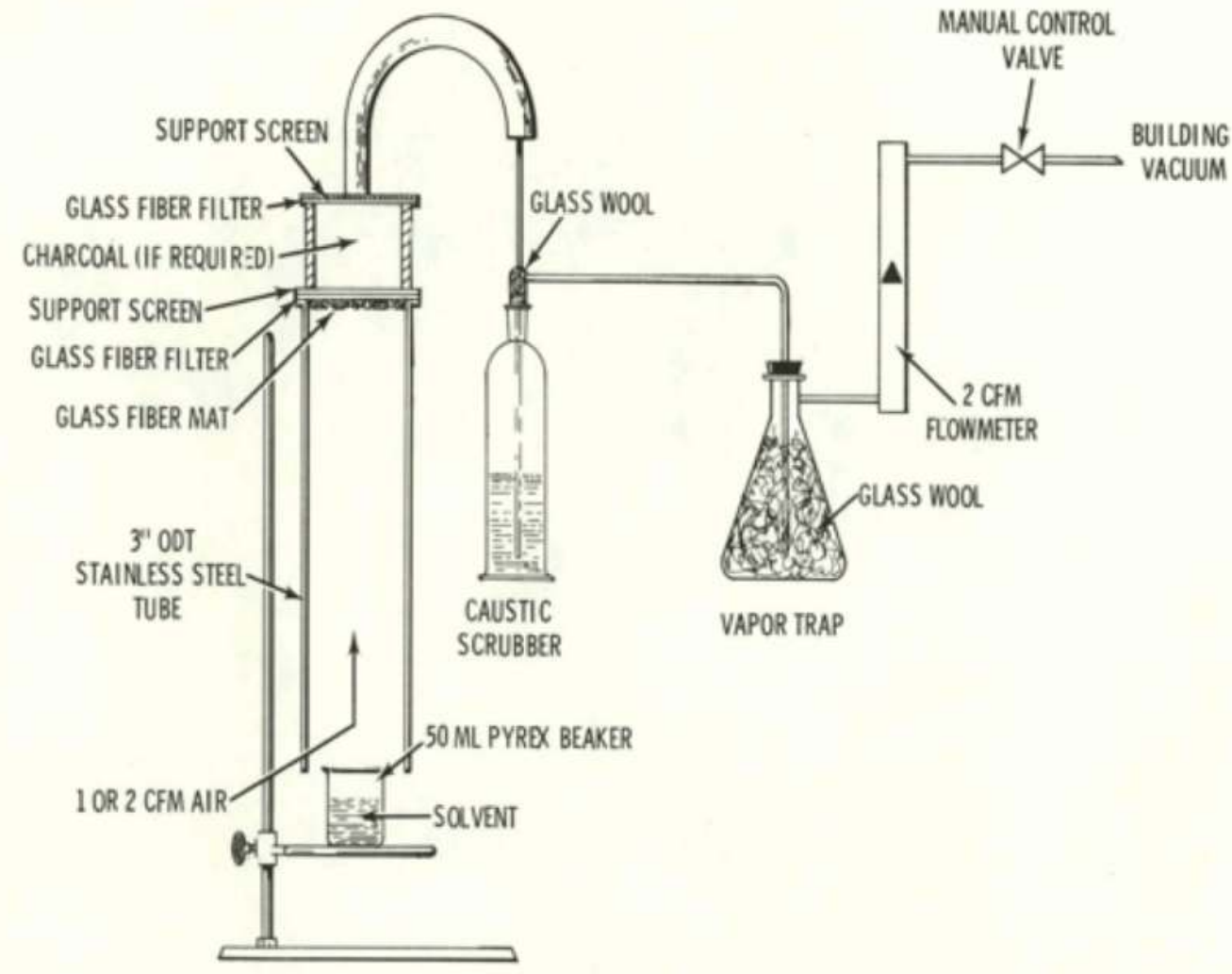




\section{WIPP radiation release has brought up old concerns about actinide aerosol behavior.}

2014 Radiological Event at the WIPP: On February 14, 2014, a radiation release occurred at the WIPP from a compromised drum of contact-handled transuranic waste emplaced underground in the WIPP facility. The drum contained nitrate salts, processed and emplaced at the WIPP in late 2013. The Department of Energy Accident Investigation Board determined the cause of the radiation release was an exothermic reaction due to the use of incompatible, organic sorbent material instead of inorganic sorbents for absorbing free liquids in sludge waste containers.
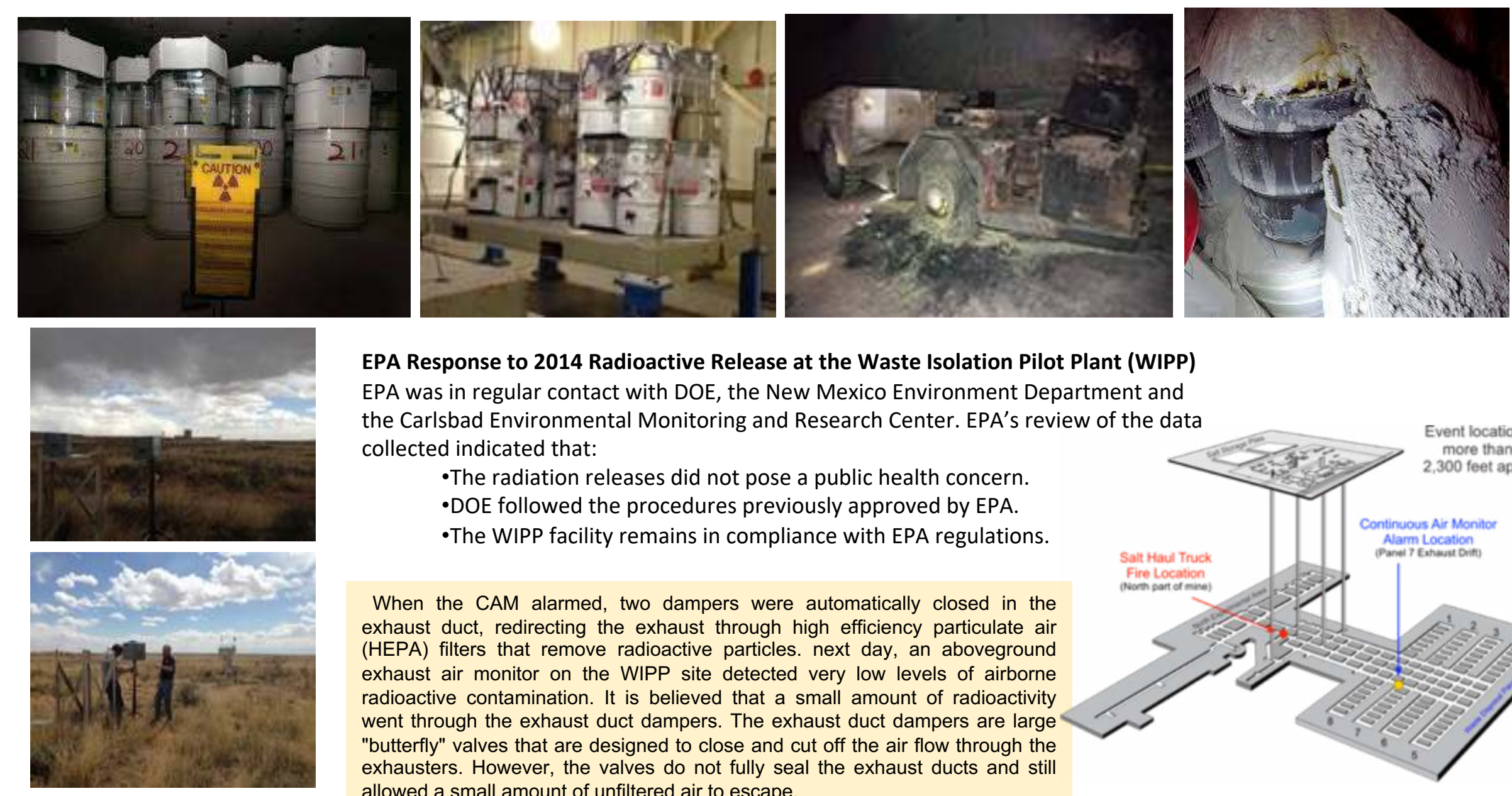

EPA Response to 2014 Radioactive Release at the Waste Isolation Pilot Plant (WIPP)

EPA was in regular contact with DOE, the New Mexico Environment Department and the Carlsbad Environmental Monitoring and Research Center. EPA's review of the data collected indicated that:

-The radiation releases did not pose a public health concern. -DOE followed the procedures previously approved by EPA.

-The WIPP facility remains in compliance with EPA regulations.

When the CAM alarmed, two dampers were automatically closed in the exhaust duct, redirecting the exhaust through high efficiency particulate air (HEPA) filters that remove radioactive particles. next day, an aboveground exhaust air monitor on the WIPP site detected very low levels of airborne radioactive contamination. It is believed that a small amount of radioactivity went through the exhaust duct dampers. The exhaust duct dampers are large "butterfly" valves that are designed to close and cut off the air flow through the exhausters. However, the valves do not fully seal the exhaust ducts and still allowed a small amount of unfiltered air to escape.

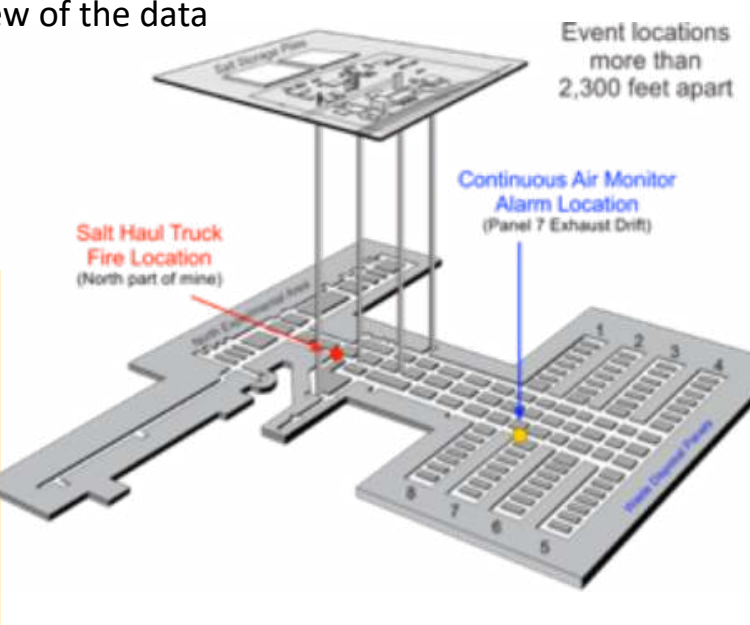




\section{Sandia's modeling efforts have supported work on the DOE Handbook $3010^{1}$}

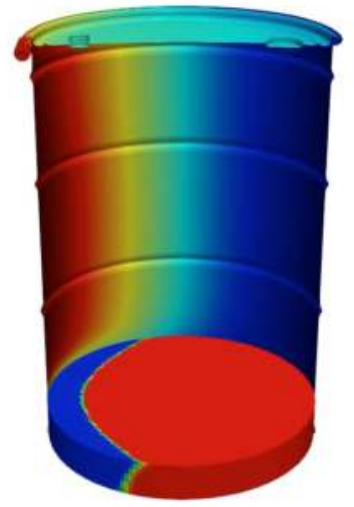

- SNL's Fire Sciences and Technology department simulated a variety of contaminant release scenarios using Computational Fluid Dynamics (SIERRA/FUEGO) tools

- Contaminant entrainment/release has been the focus of previous studies:

- Benchtop ("beaker") fires

- Gasoline fuel fires

- Drum fires

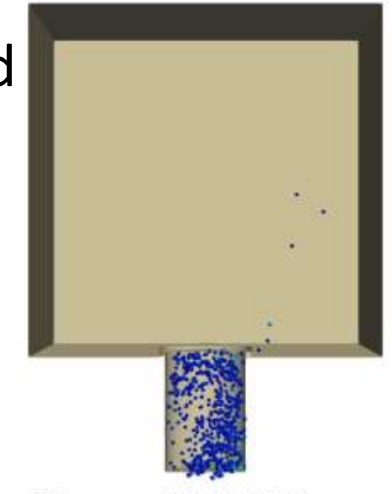

Time: $510.00 \mathrm{sec}$
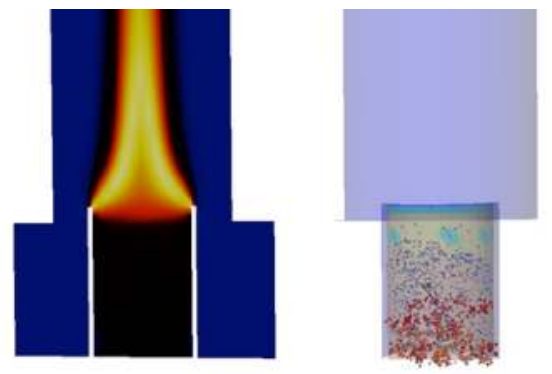

60.000 sec. Deposition Number Density $1 / \mathrm{m} \wedge 2$ $10+4$

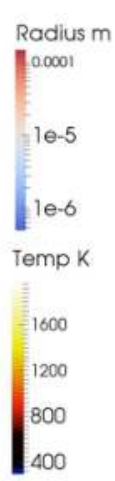

- Beaker fire experimental effort modeling a solvent heated to boiling point and ignited

- Focused primarily on $d-U$ release from kerosene with $30 \%$ TBP.

- Experimental repetition appear challenging

- Code enhancements were made to better track composition of entrained particles

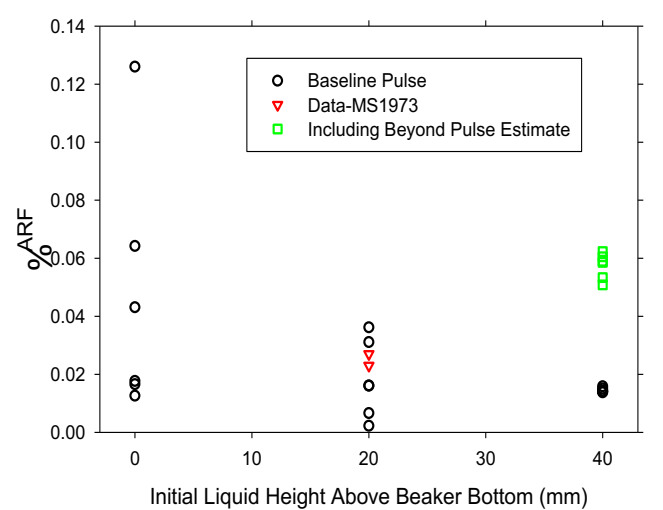

Initial Liquid Height Above Beaker Bottom (mm)

Brown, A. L., and Louie, D. L. Y., "Contaminant Entrainment in a Liquid Fuel Fire," TFESC-12948, 1st Thermal and Fluid Engineering Summer Conference, New York, NY, August 9-12, 2015. Brown, A. L., Zepper, E. T., Louie, D. L. Y., and Restrepo, L. “Contaminant Entrainment from a Gasoline Pool Fire," SAND2015-7185C, September 2015, Sandia National Laboratories.

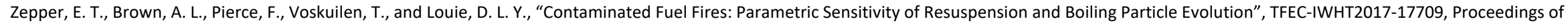
the $2^{\text {nd }}$ Thermal and Fluid Engineering Conference, Las Vegas, NV, April 2-5, 2017. 


\section{Computational modeling of flame aerosol production lead to the design of the burn chamber.}
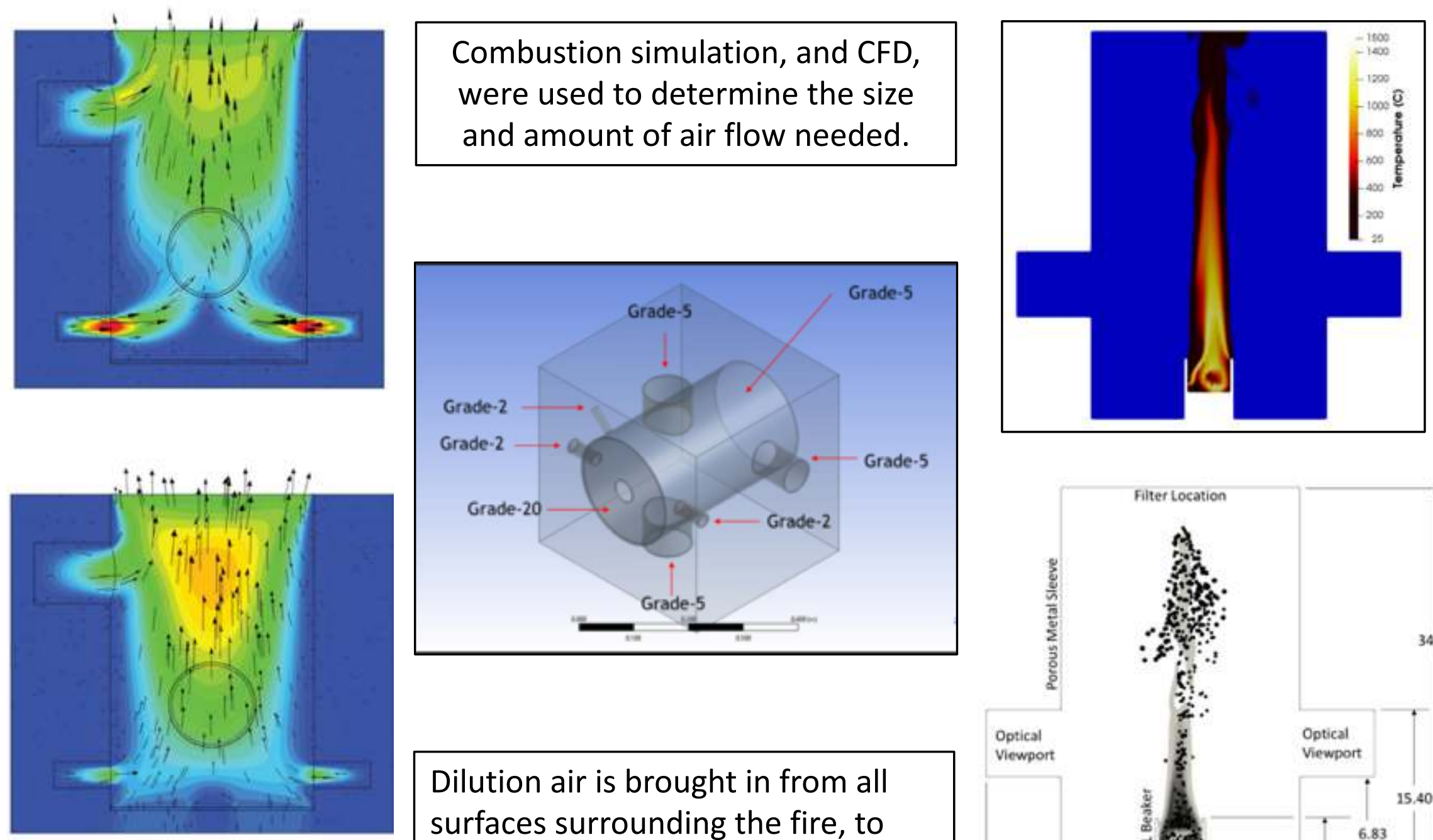

Dilution air is brought in from all surfaces surrounding the fire, to sweep particulate up to the filter.

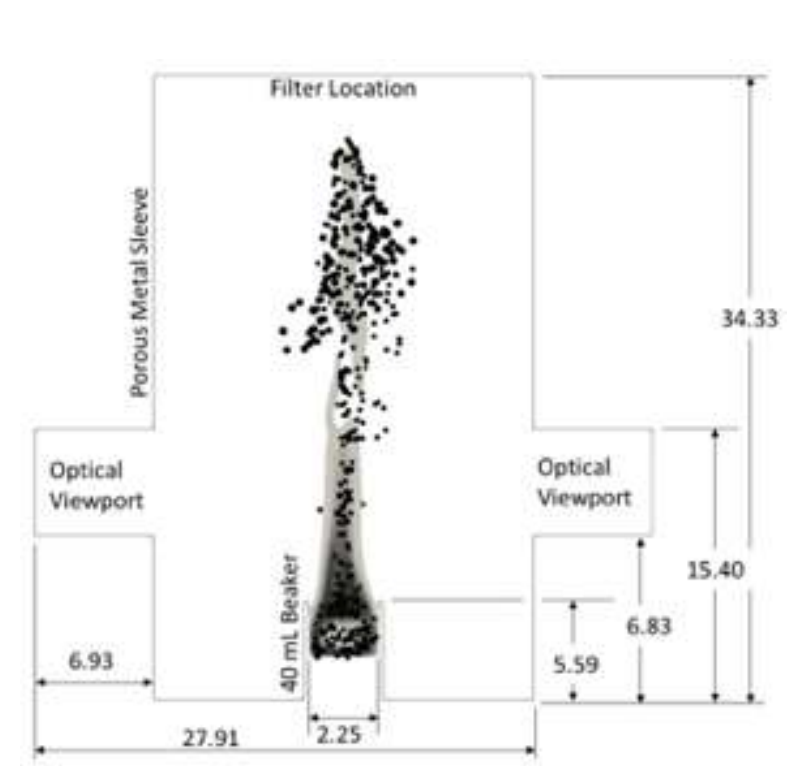


Can surrogate modeling (e.g., drum fires), extend the results to the An-contaminant (d-U), aiding in safety assertions?
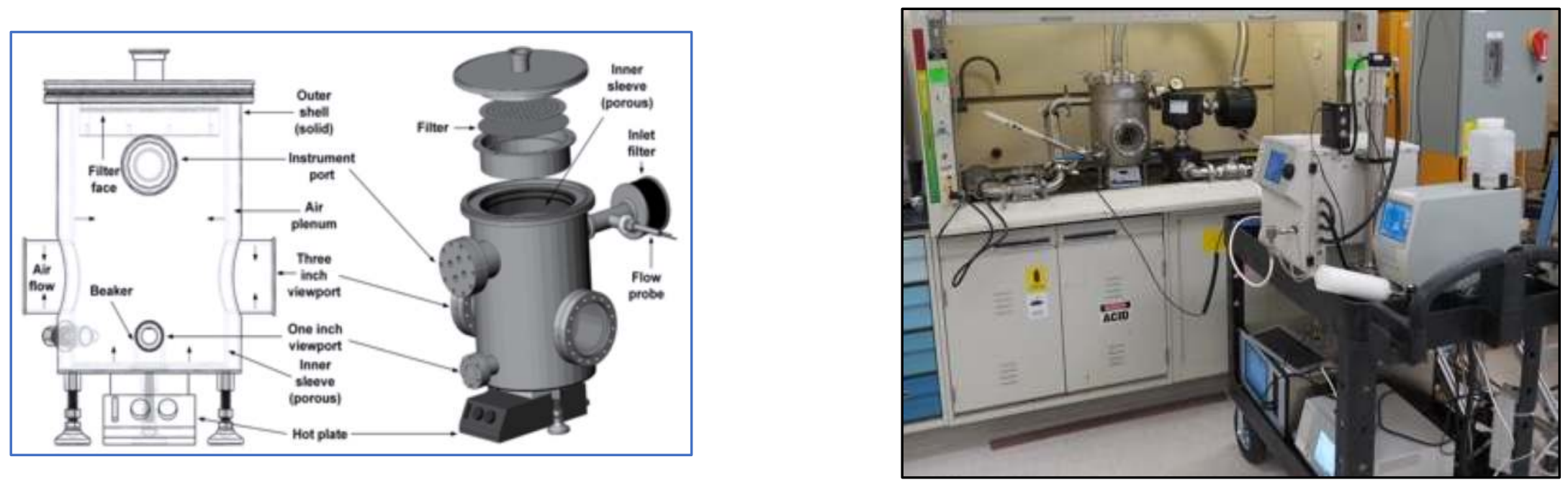

- Tests were conducted in order to use extractive sampling techniques in order to characterize particles close to real-time

- Tests were conducted where all particulate was captured on the filter at the top of the chamber and filters were analyzed destructively with ICP-MS (ARF)

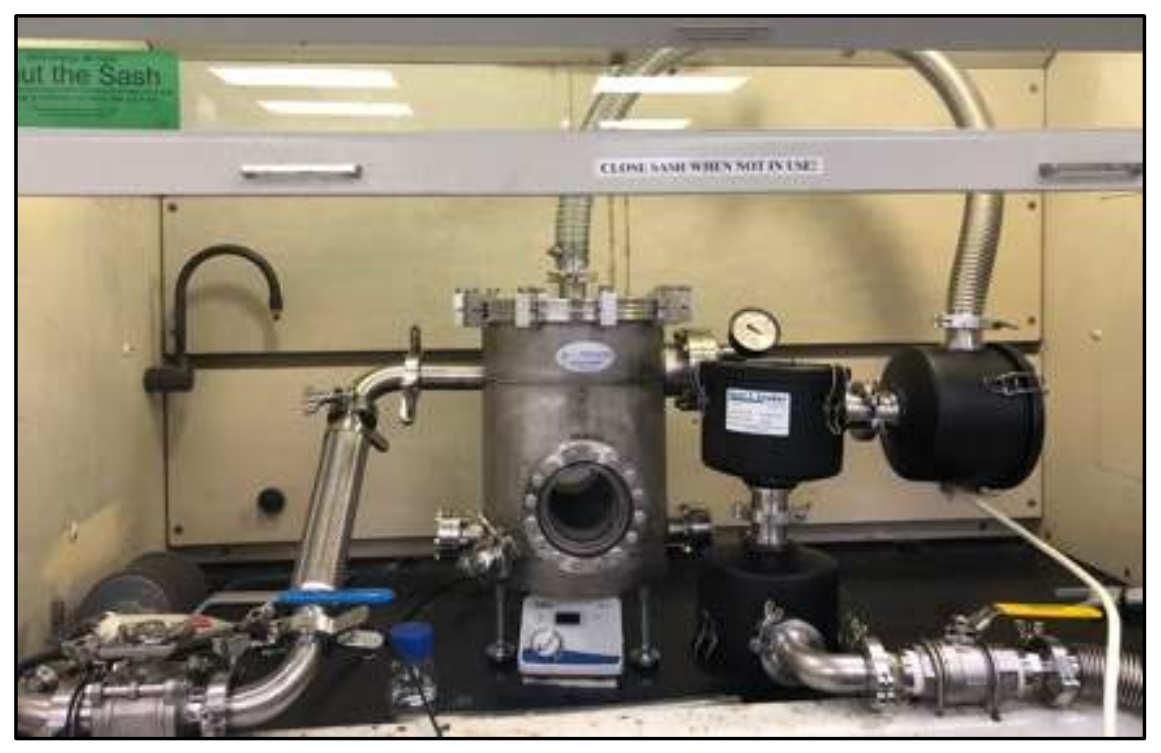


The ideal simulant for aerosol particulate have led (not surprising) to the Ln cations, but favor late Ln.

- $\quad$ Simulating experiments conducted in 1973 by Mishima and Schwendiman

- DOE Handbook 3010 Section 3.3.1 "Burning of Small Volume/Surface Area 30\% TBP-Kerosene Solutions, No Vigorous Boil-off"

- Process solution from PUREX liquid-liquid extraction, actinides, kerosene, tri-butyl phosphate (plus fission products)

- Ideal simulant

- Density of resultant particle similar (nitrate vs. oxide), does the particle get hot enough to undergo calcination?

- Solubility of surrogate metal similar to solubility of uranium ( 120 grams per liter).

- Concentration of metal in solution will have a substantial effect on resultant particle size.

- Ideal simulant depends on the process being simulated (e.g., PUREX waste, metal fabrication, spent fuel)

- Focused on TBP (or TPhP)/kerosene experiments.

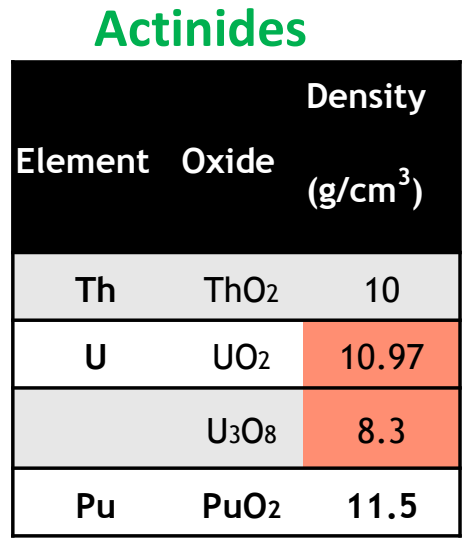

Transition metals

\begin{tabular}{ccc|}
\hline Element Oxide & $\begin{array}{c}\text { Density } \\
\left(\mathrm{g} / \mathrm{cm}^{3}\right)\end{array}$ \\
& $\mathrm{WO}_{2}$ & 10.8 \\
\hline $\mathrm{W}$ & $\mathrm{WO}_{3}$ & 7.16 \\
\hline & $\mathrm{Bi}_{2} \mathrm{O}_{3}$ & 8.9 \\
\hline $\mathrm{Bi}$ & $\mathrm{HfO}_{2}$ & 9.68 \\
\hline
\end{tabular}

\begin{tabular}{|ccc|}
\hline Element & Oxide & $\begin{array}{c}\text { Density } \\
\left(\mathbf{g} / \mathbf{c m}^{3}\right.\end{array}$ \\
\hline $\mathrm{La}$ & $\mathrm{La}_{2} \mathrm{O}_{3}$ & 6.51 \\
\hline $\mathrm{Ce}$ & $\mathrm{Ce}_{2} \mathrm{O}_{3}$ & 6.2 \\
\hline & $\mathrm{CeO}_{2}$ & 7.215 \\
\hline $\mathrm{Pr}$ & $\mathrm{Pr}_{2} \mathrm{O}_{3}$ & 6.9 \\
\hline & $\mathrm{Pr}_{6} \mathrm{O}_{11}$ & 6 \\
\hline $\mathrm{Nd}$ & $\mathrm{Nd}_{2} \mathrm{O}_{3}$ & 7.24 \\
\hline $\mathrm{Pm}$ & $\mathrm{Pm}_{2} \mathrm{O}_{3}$ & 6.85 \\
\hline & $\mathrm{Pm}_{2} \mathrm{O}_{3}$ & 7.48 \\
\hline & $\mathrm{Pm}_{2} \mathrm{O}_{3}$ & 7.62 \\
\hline $\mathrm{Sm}$ & $\mathrm{Sm}_{2} \mathrm{O}_{3}$ & 8.347 \\
\hline $\mathrm{Eu}$ & $\mathrm{Eu}_{2} \mathrm{O}_{3}$ & 7.42 \\
\hline & $\mathrm{Eu}_{2} \mathrm{O}_{3}$ & 7.42 \\
\hline $\mathrm{Gd}$ & $\mathrm{Gd}_{2} \mathrm{O}_{3}$ & 7.07 \\
\hline $\mathrm{Tb}$ & $\mathrm{Tb}_{2} \mathrm{O}_{3}$ & 7.91 \\
\hline & $\mathrm{Tb}_{4} \mathrm{O}_{7}$ & 7.3 \\
\hline $\mathrm{Dy}$ & $\mathrm{Dy}_{2} \mathrm{O}_{3}$ & 7.8 \\
\hline $\mathrm{Ho}$ & $\mathrm{Ho}_{2} \mathrm{O}_{3}$ & 8.1 \\
\hline $\mathrm{Er}$ & $\mathrm{Er}_{2} \mathrm{O}_{3}$ & 8.64 \\
\hline $\mathrm{Tm}$ & $\mathrm{Tm}_{2} \mathrm{O}_{3}$ & 8.6 \\
\hline $\mathrm{Yb}$ & $\mathrm{Yb}_{2} \mathrm{O}_{3}$ & 9.17 \\
\hline $\mathrm{Lu}$ & $\mathrm{Lu}_{2} \mathrm{O}_{3}$ & 9.42 \\
\hline & & \\
\hline
\end{tabular}
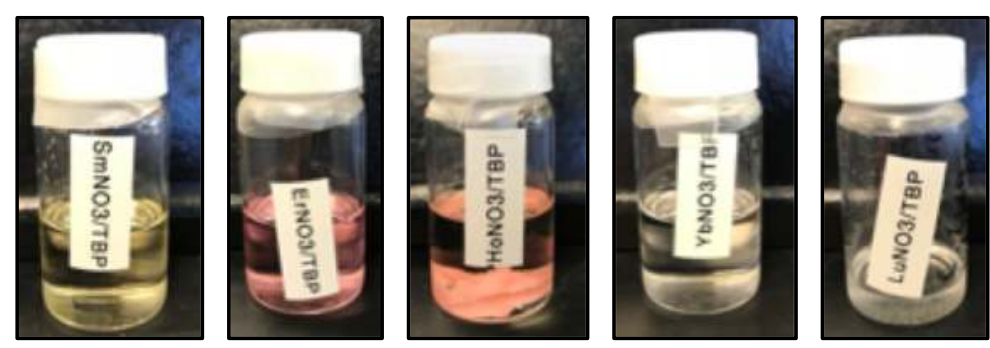

a $30 \%$ organophosphate/kerosene solution.

$$
\left[\mathrm{M}\left[\left(\mathrm{NO}_{3}\right)_{3} \cdot n \mathrm{H}_{2} \mathrm{O}\right]+(\mathrm{xs}) \mathrm{O}=\mathrm{P}(\mathrm{OR})_{3}\right.
$$
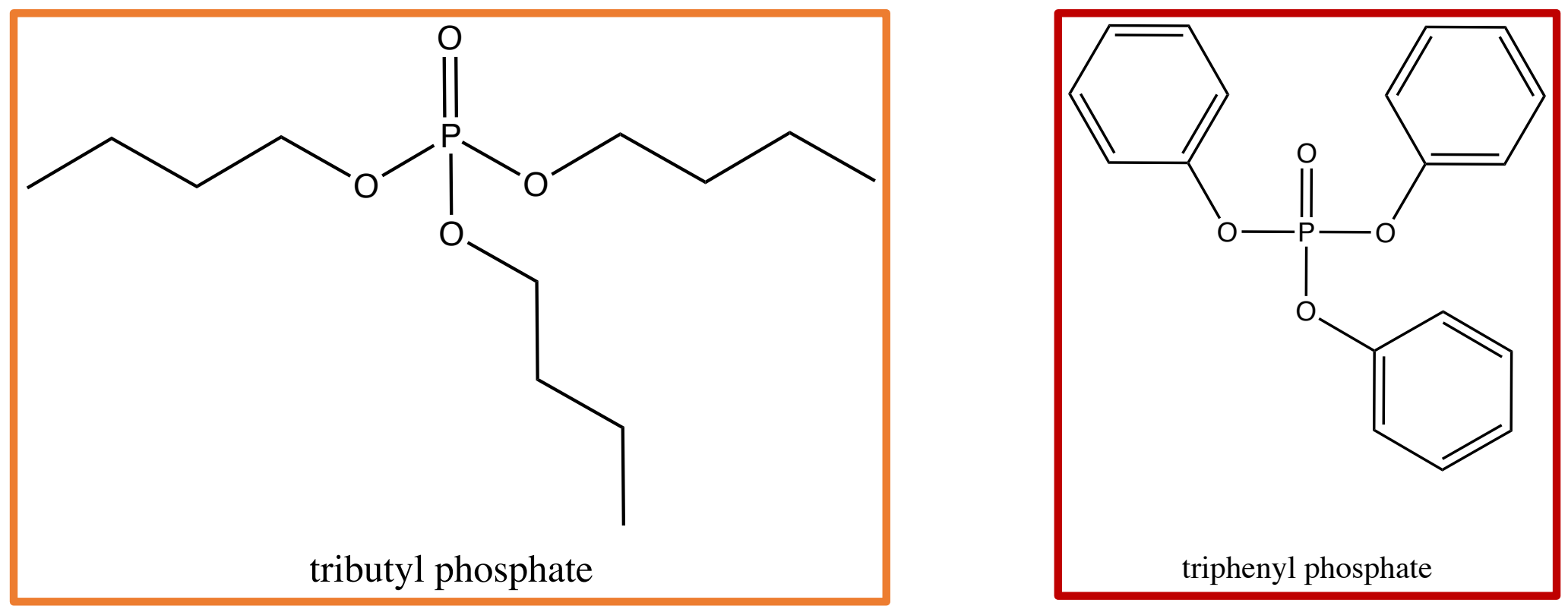

TBP

TPhP 


\section{Literature uranyl nitrate organophosphate structures reported are monomeric or dimeric.}

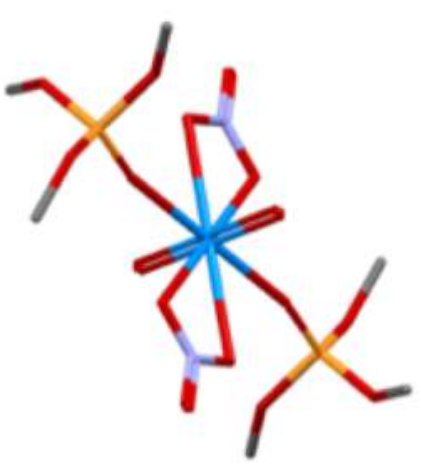

BIZXUD: Uranyl nitrate trimethylphosphate Agostini et al. ICA, 62 (1982) 237. DOI: $10.1016 /$ S0020-1693(00)88507-2

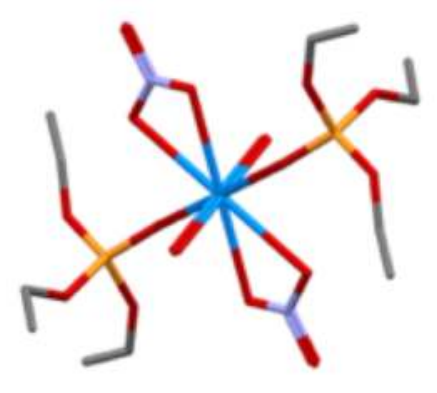

UNOEPO: Uranyl nitrate triethylphosphate Fleming and Lynton Chem. Ind. (1960) 1415. UNOEPO01: Kanellakopulos et al. Z. Anorg. Allg Chem. 619 (1993) 593.

DOI: 10.1002/zaac.19936190327

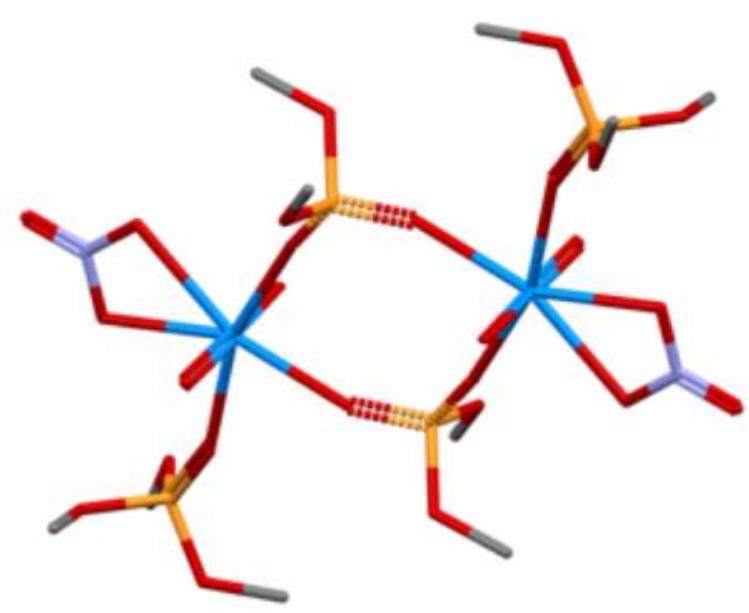

LIDTEZ: Uranyl nitrate bis(trimethylphosphate) Charushnikova et al. Radiochim Acta, (2012) 173. DOI: 10.1524/ract.2012.1899

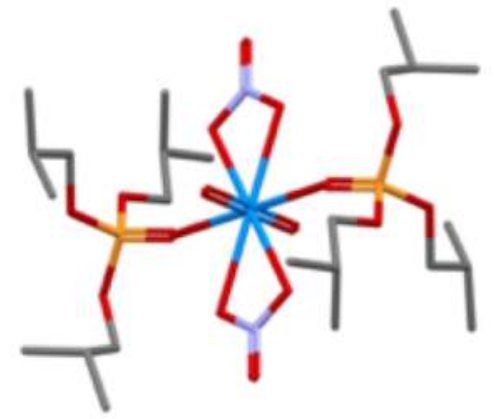

DEFMEG: Uranyl nitrate triisobutylphosphate Burns et al. Acta Cryst. C 41 (1985) 1446. DOI: $10.1107 /$ S0108270185008125

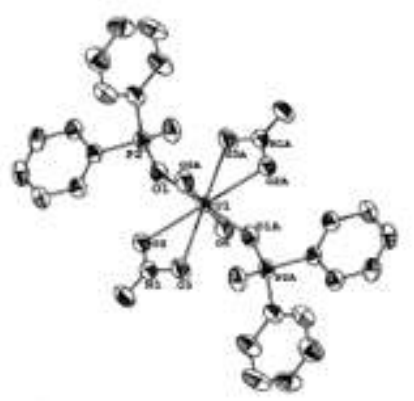

Uranyl nitrate methyldiphenyl phosphine oxide Martin et al. J. Nucl. Sci. Tech. (2002) 453-456 DOI: 10.1080/00223131.2002.10875505

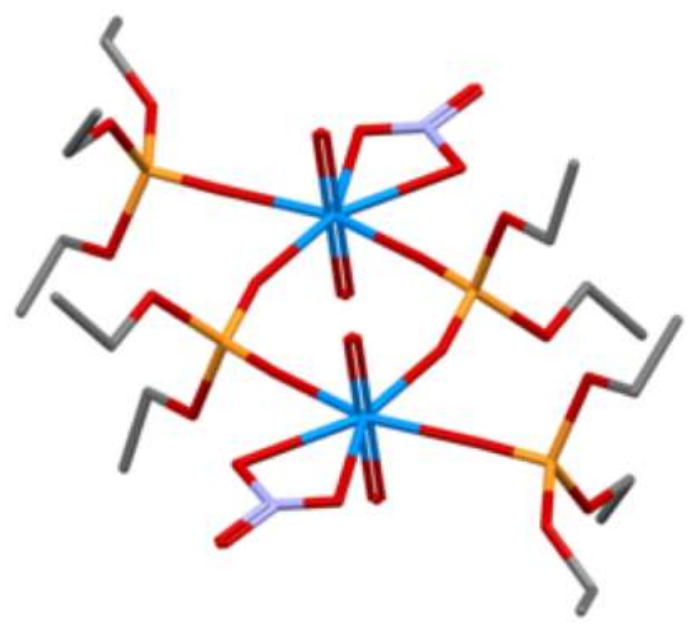

WEWXAX: Uranyl nitrate bis(triethylphosphate)

Kanellakopulos et al. Z. Anorg. Allg. Chem. (1993) 593.

DOI: 10.1002/zaac.19936190327

WEWXAX01: Charushnikova et al. Radiochim. Acta (2012) 173. 
Single crystal X-ray diffraction reveals for U-TPhP a monomer $\mathrm{w} / \mathrm{two}$ phosphate, two nitrates, two oxides.

$$
\begin{gathered}
\mathrm{U}^{0}+(\mathrm{aq}) \mathrm{HNO}_{3} \\
{\left[\mathrm{U}(\mathrm{O})_{2}\left(\mathrm{NO}_{3}\right)_{2} \bullet 6 \mathrm{H}_{2} \mathrm{O}\right]+(\mathrm{xs}) \mathrm{O}=\mathrm{P}(\mathrm{OR})_{3}}
\end{gathered}
$$
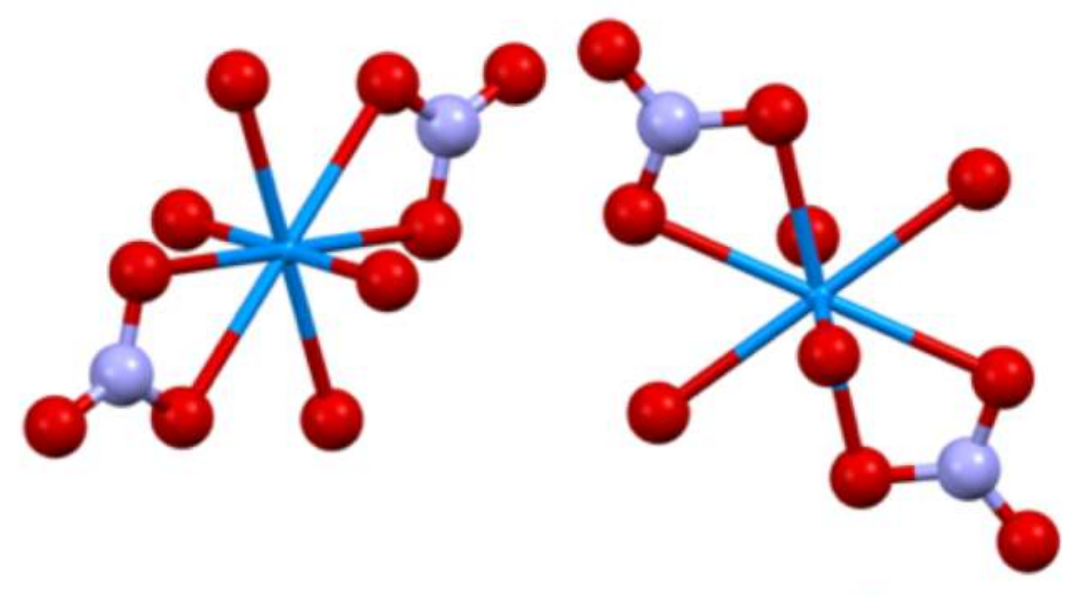

○

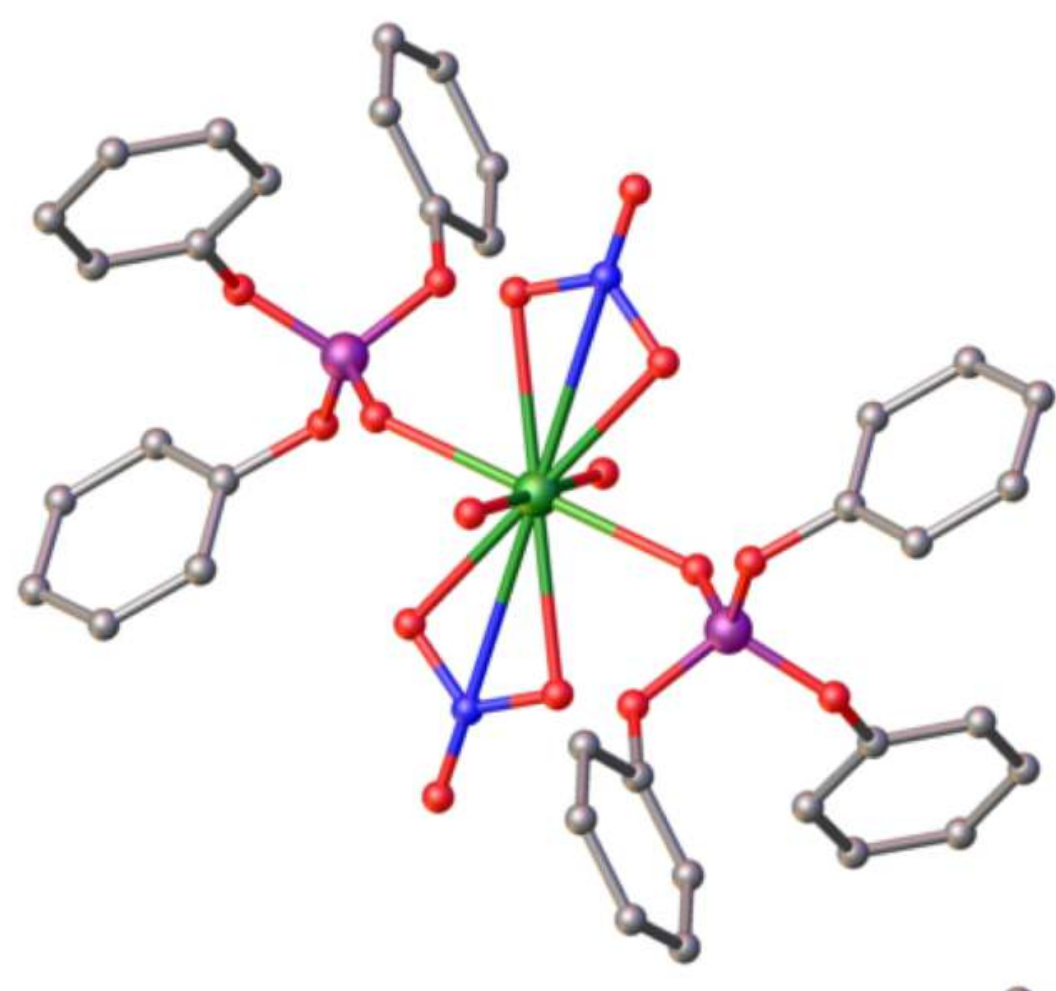

$2\left[\mathrm{U}(\mathrm{O})_{2}\left(\mathrm{NO}_{3}\right)_{2}\left(\mathrm{H}_{2} \mathrm{O}\right)_{2}\right] \bullet \mathrm{H}_{2} \mathrm{O}$

$\left[\mathrm{U}(\mathrm{O})_{2}\left(\mathrm{NO}_{3}\right)_{2}(\mathrm{TPhP})_{2}\right]$ 
Understanding the lanthanide nitrate hydrates dissolved in a $30 \%$ organophosphate/kerosene solution was of interest.

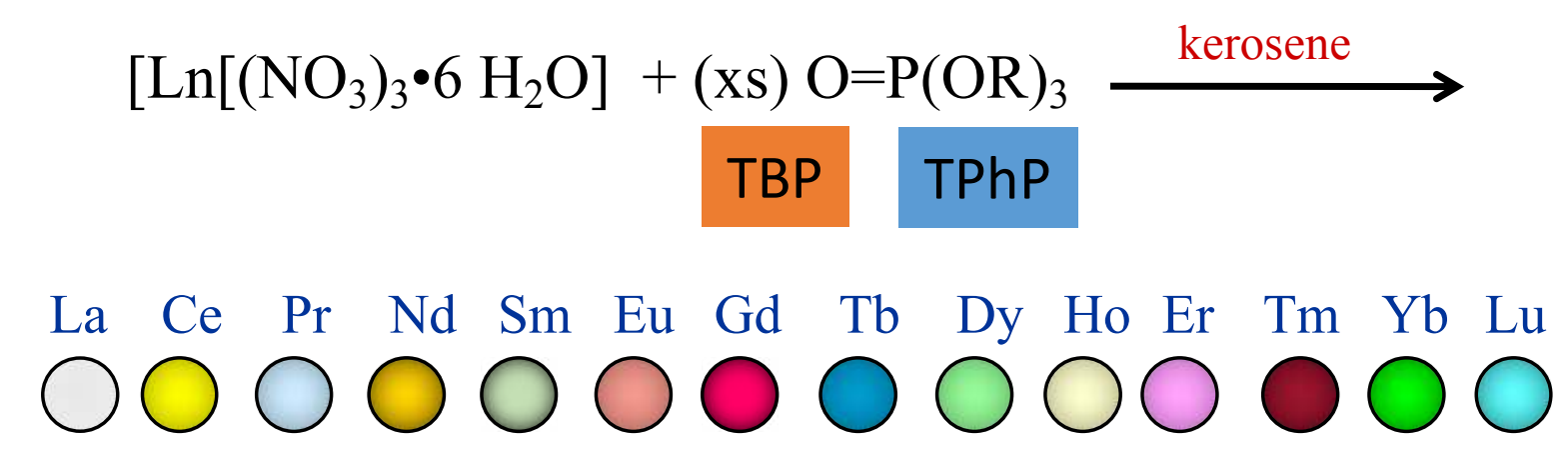

A dozen $\mathrm{Ln}-\mathrm{OP}(\mathrm{OR})_{3}$ structures reported but only one is a nitrate!

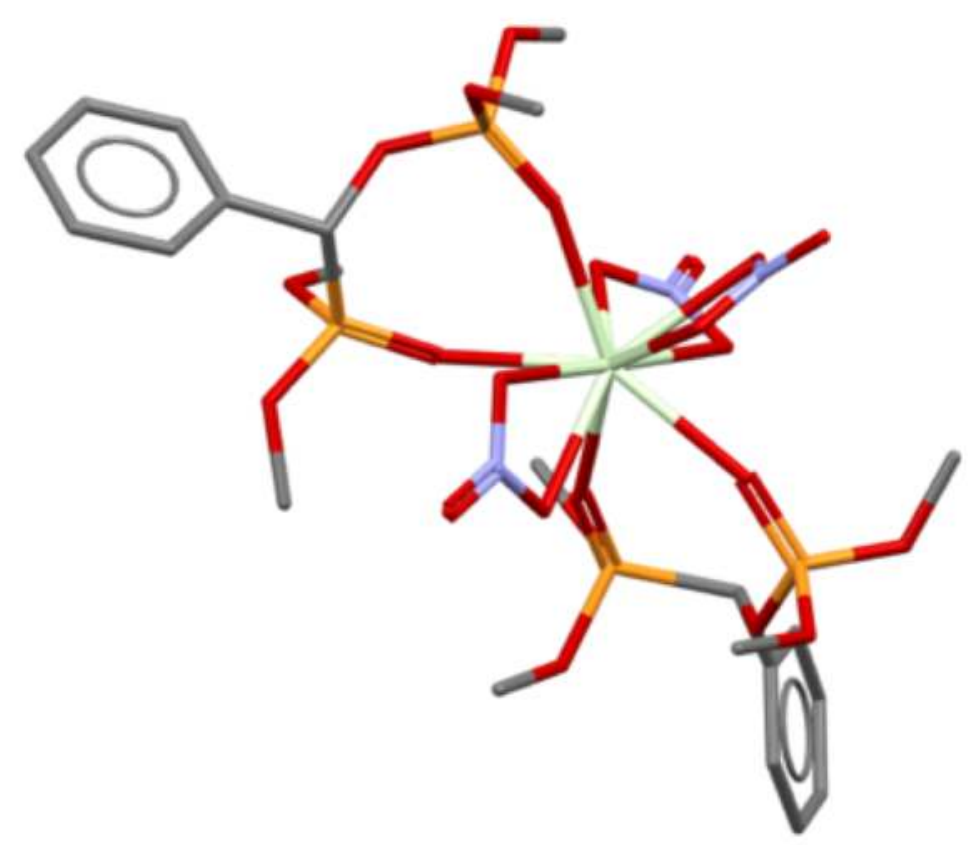

POPJAF: Praseodymium tris(nitrato-O,O')-bis(bis(dimethylphosphonato)phenylmethanol) 
Single crystal X-ray diffraction reveals for Ln-TPhP system monomers $\mathrm{w} /$ three phosphate and three nitrates isolated . .

$$
\left[\mathrm{Ln}\left[\left(\mathrm{NO}_{3}\right)_{3} \cdot 6 \mathrm{H}_{2} \mathrm{O}\right]+(\mathrm{xs}) \mathrm{O}=\mathrm{P}(\mathrm{OR})_{3} \stackrel{\text { kerosene }}{\longrightarrow} \underset{\text { acetone }}{\longrightarrow}\right.
$$
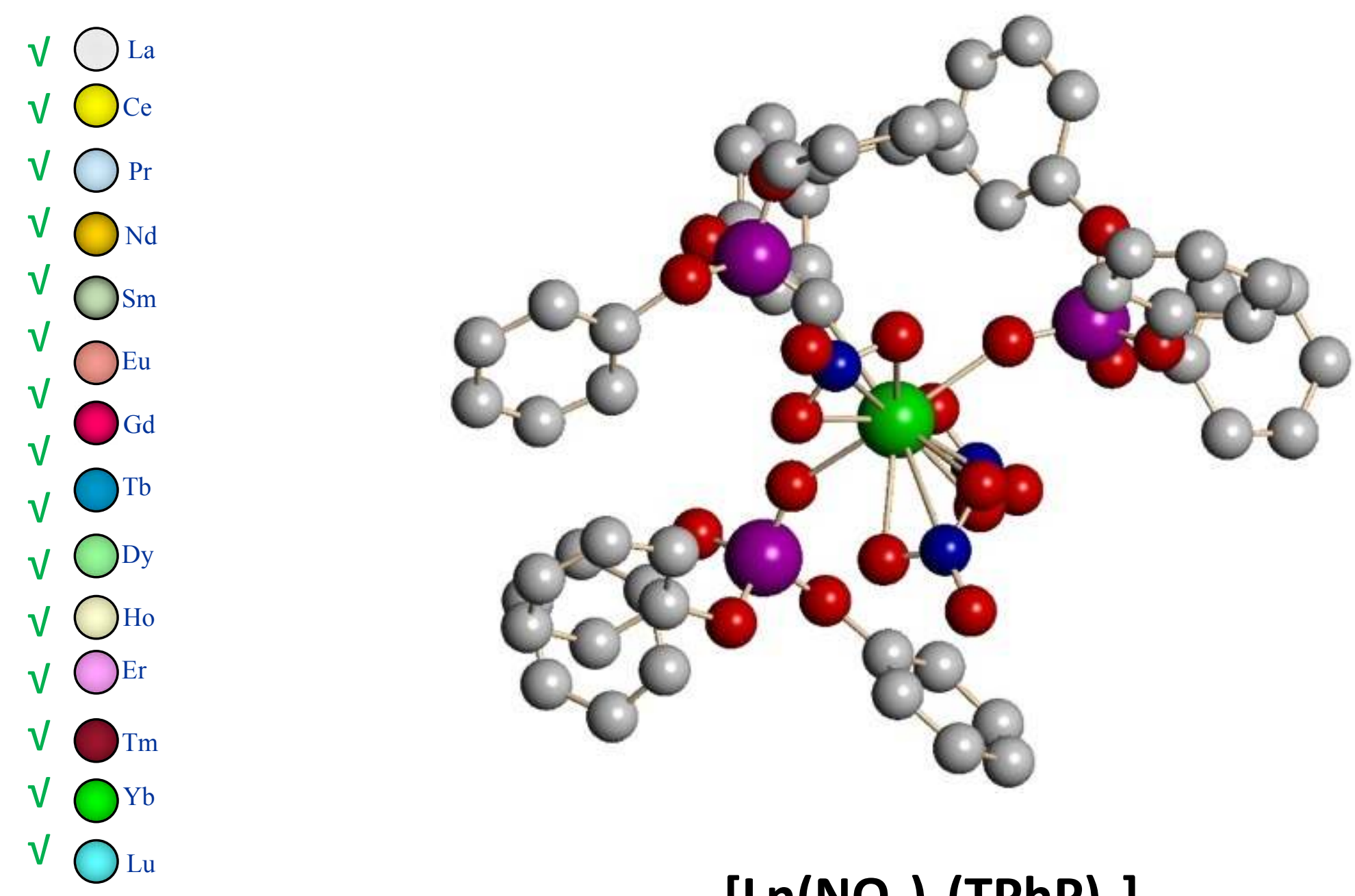

$\left[\operatorname{Ln}\left(\mathrm{NO}_{3}\right)_{3}(\mathrm{TPhP})_{3}\right]$ 
.. when crystallization from acetone a different product was isolated, where acetone was reduced to H-DAA.

$$
\left[\mathrm{Ln}\left[\left(\mathrm{NO}_{3}\right)_{3} \cdot 6 \mathrm{H}_{2} \mathrm{O}\right] \stackrel{\text { acetone }}{\longrightarrow}\right.
$$

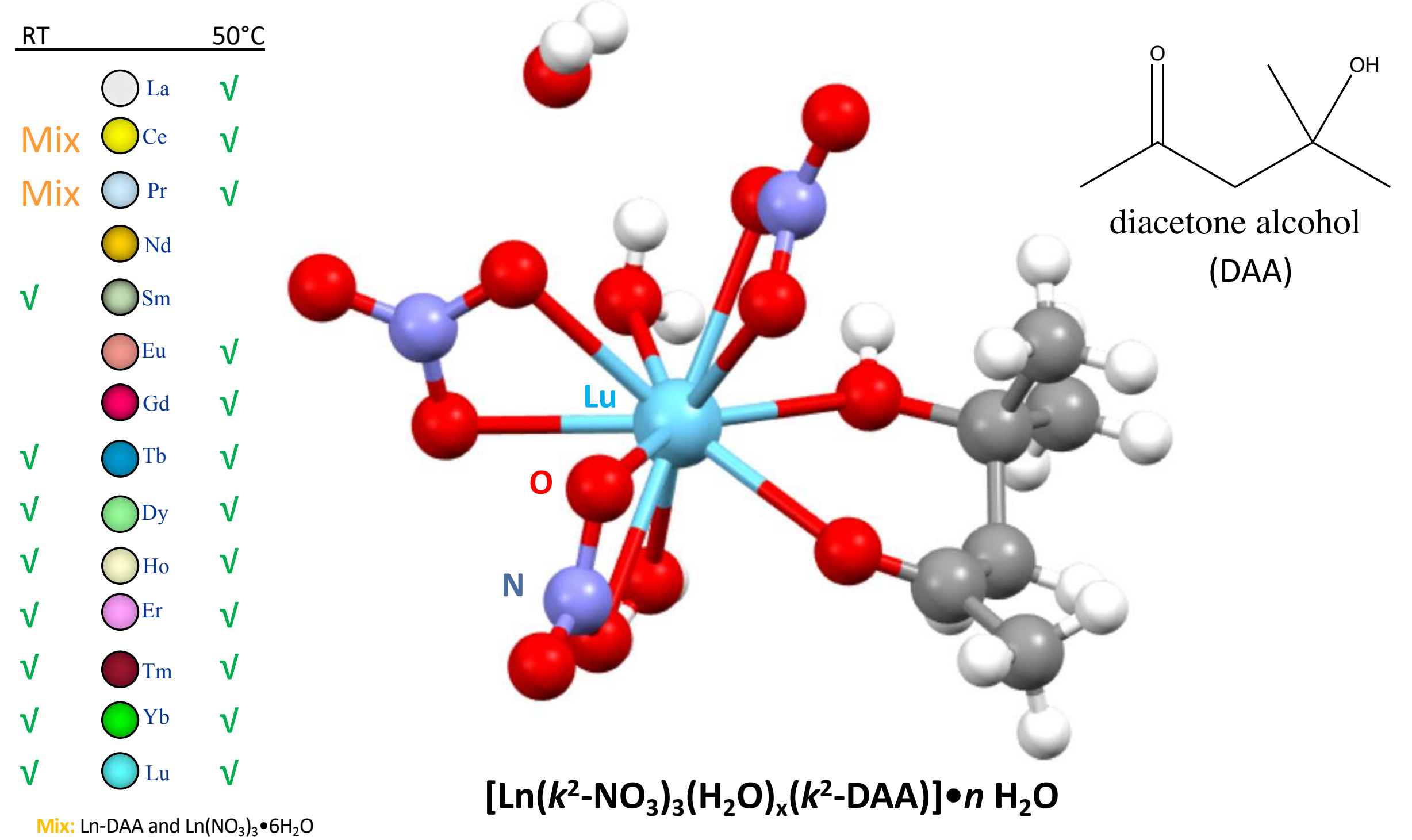


Ln-DAA-like structures reported: 1 from reduction of acetone to form the alkoxide; others H-DAA coordination

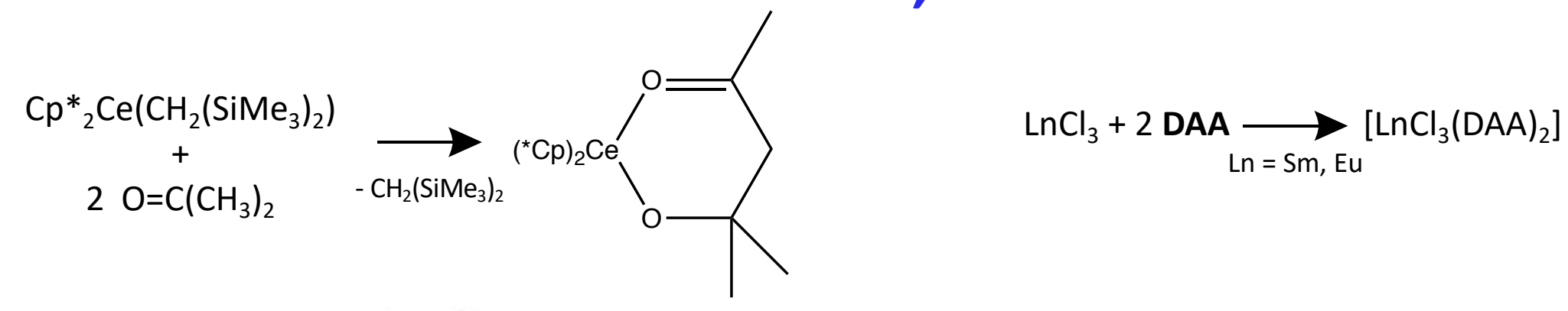

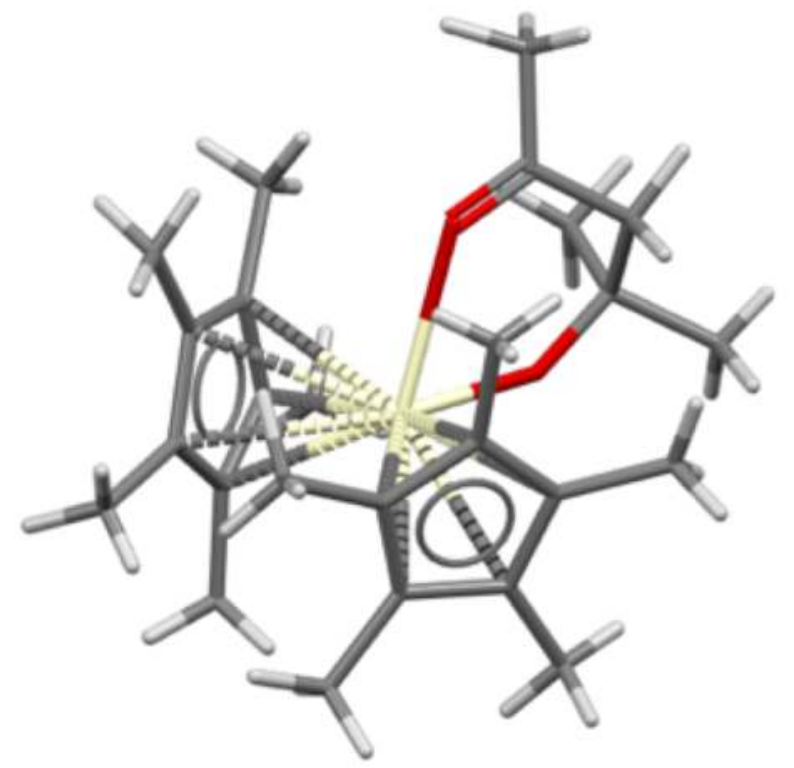

JOLYAK: Cerium bis(h5-Pentamethylcyclopentadienyl)-(4-hydroxy-4-methyl-2-pentanonato$\left.0,0^{\prime}\right)$

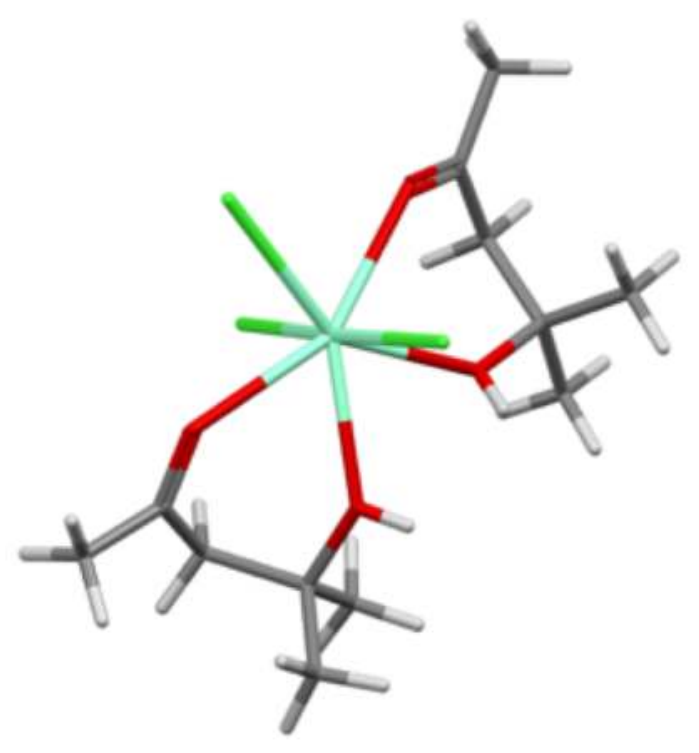

OFULEG: Samarium trichloro bis(4-Hydroxy-4-methyl-2-pentanone)

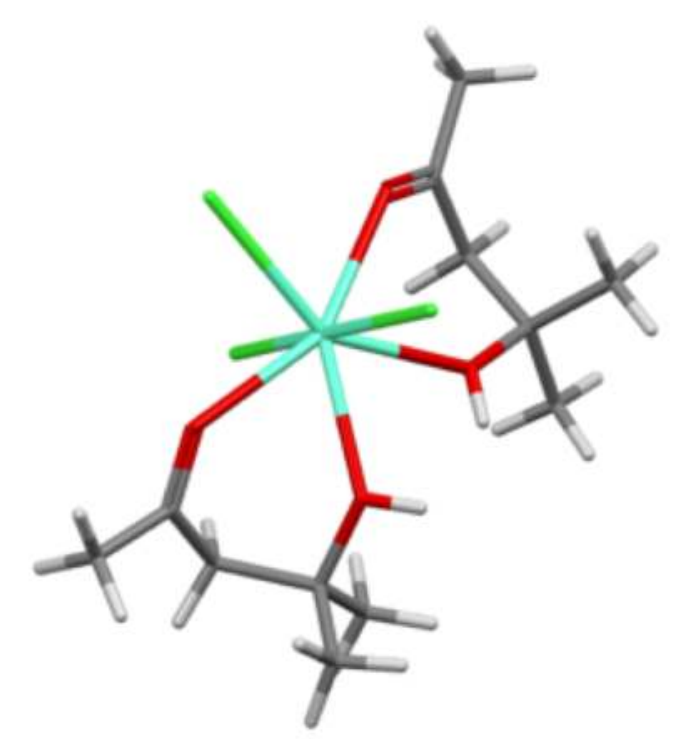

OFULEG: Europium trichloro bis(4-Hydroxy-4-methyl-2-pentanone) 


\section{Most reports on DAA from $\mathrm{O}=\mathrm{C}\left(\mathrm{CH}_{3}\right)_{2}$ requires strong base}

Diacetone alcohol can be prepared from acetone by the Soxhlet extraction of acetone using alkali ( $\mathrm{NaOH}$ or $\mathrm{KOH})$ and alkaline earth metal hydroxides[1]: calcium hydroxide [2] < barium hydroxides[3].
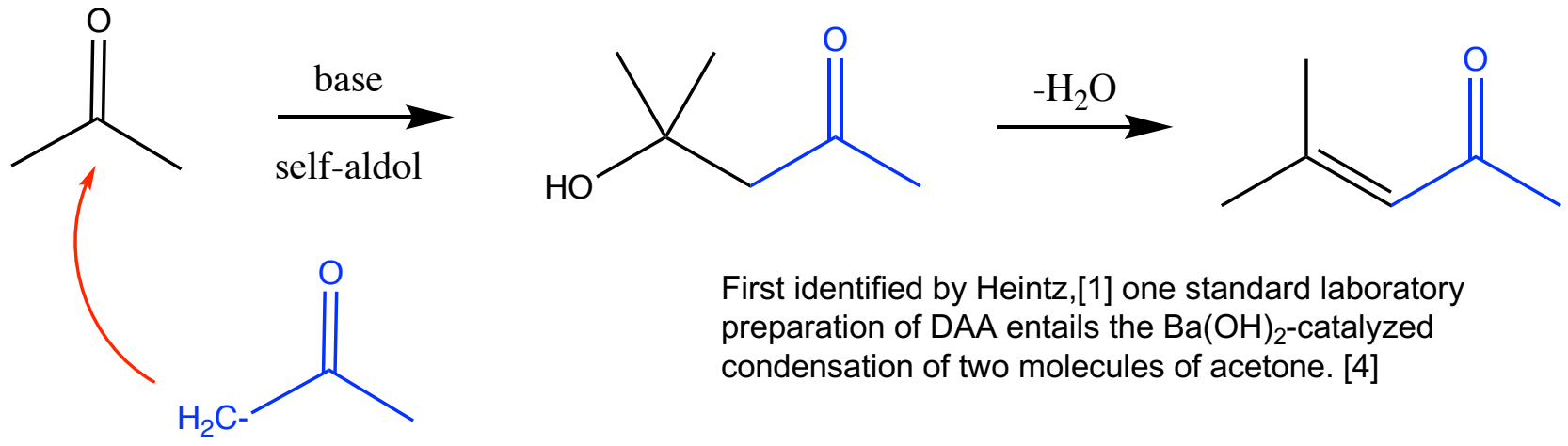

First identified by Heintz,[1] one standard laboratory preparation of DAA entails the $\mathrm{Ba}(\mathrm{OH})_{2}$-catalyzed condensation of two molecules of acetone. [4]

Diacetone alcohol (DAA) is mainly used as a solvent in industrial and professional applications in coatings, cleaning, oil and gas filed drilling, lubricants, metal working fluids, polymer processing and water treatment. It is found in a number of consumer products such as adhesives, inks, paints, household cleaners and agrochemicals. The substance is also a chemical intermediate for the preparation of other compounds. It is used in cellulose ester lacquers, particularly of the brushing type, where it produces brilliant gloss and hard film and where its lack of odor is desirable. It is used in lacquer thinners, dopes, wood stains, wood preservatives and printing pastes; in coating compositions for paper and textiles; permanent markers; in making artificial silk and leather; in imitation gold leaf; in celluloid cements; as a preservative for animal tissue; in metal cleaning compounds; in the manufacture of photographic film; and in hydraulic brake fluids. https://en.wikipedia.org/wiki/Diacetone_alcohol

US20170158591A1 "Synthesis of diacetone alcohol and meistyl oxide"

Disclosed herein are processes for synthesizing diacetone alcohol. In some embodiments, an exemplary process for synthesizing diacetone alcohol includes contacting acetone with a neutral heterogeneous catalyst (metal aluminosilicates) at a temperature of less than $100^{\circ} \mathrm{C}$ $\left(20^{\circ} \mathrm{C}\right.$. to about $\left.25^{\circ} \mathrm{C}\right)$, to provide diacetone alcohol. [5]

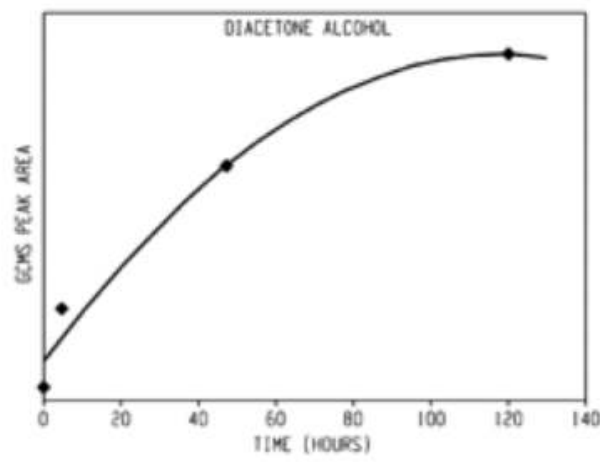

Fig. 2
FIG. 2 is a graphical representation of formation of DAA from acetone over time according to an exemplary process of the present disclosure.

1. Heintz, Ann. 169, 114 (1873); Locquin, Ann. Chim. (9) 19, 32 (1923); Thomson, U. S. pat. 1,654,103 [C. A. 22, 787 (1928)]; Ellis, U. S. pat. 1,701,473 [C. A. 23, 1419 (1929)].

2. Hoffman, Ger. pat. 229,678 [Frdl. 10, 998 (1910-12)]; U. S. pat. 1,082,424 [C. A. 8, 788 (1914)]; Edmonds, U. S. pat. 1,550,792 [C. A. 20, 51 (1926)].

3. Kyriakides, J. Am. Soc. 36, 534 (1914).

4. Conant, J. B.; Tuttle, N. (1921). "Diacetone Alcohol". Organic Syntheses. 1: 45. doi:10.15227/orgsyn.001.0045.

5. Osei-Twum et al, U.S. pat US20170158591A1 “Synthesis of diacetone alcohol and meistyl oxide" Jun. 8, 2017. 


\section{Proposed acid catalyzed reduction of $\mathrm{O}=\mathrm{C}\left(\mathrm{CH}_{3}\right)_{2}$ by $\mathrm{Ln}\left(\mathrm{NO}_{3}\right)_{3} \cdot 6 \mathrm{H}_{2} \mathrm{O}$}

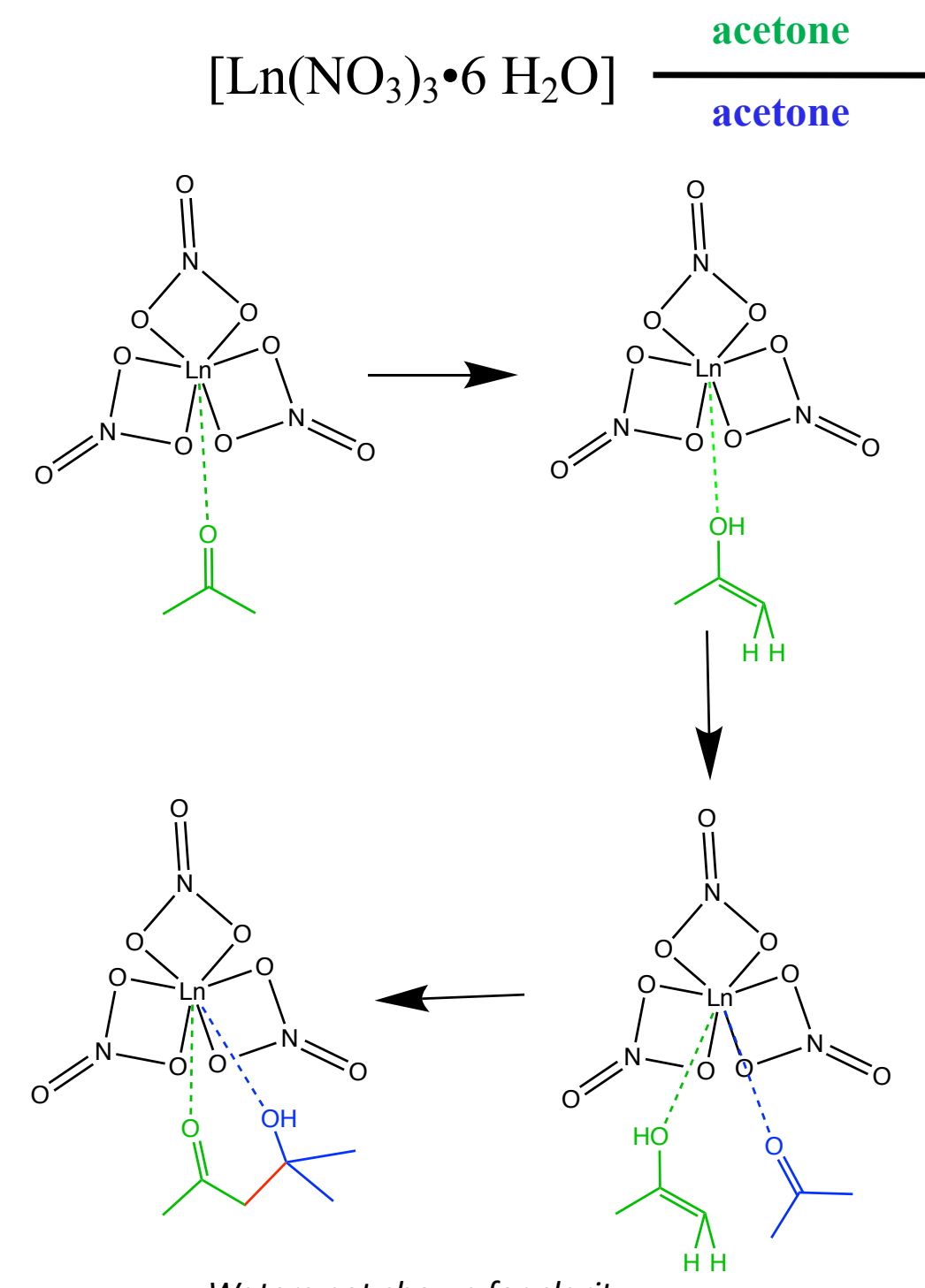

$\left[\mathrm{Ln}\left(\mathrm{NO}_{3}\right)_{3}(\mathrm{DAA})\right]$

Waters not shown for clarity

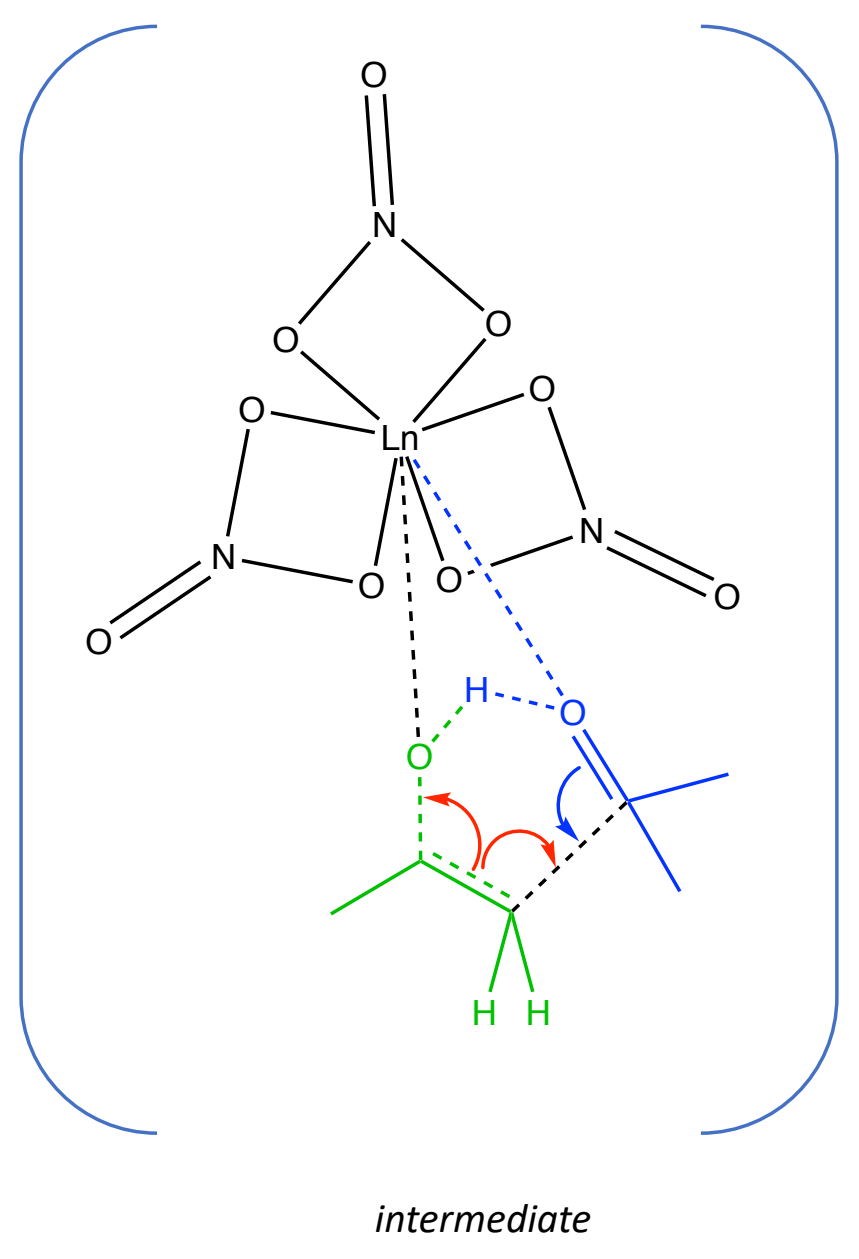


Single crystal X-ray diffraction reveals for Ln-TPhP system monomers $\mathrm{w} /$ three phosphate and three nitrates isolated.

$$
\left[\mathrm{Ln}\left[\left(\mathrm{NO}_{3}\right)_{3} \cdot 6 \mathrm{H}_{2} \mathrm{O}\right]+(\mathrm{xs}) \mathrm{O}=\mathrm{P}(\mathrm{OR})_{3} \stackrel{\text { kerosene }}{\longrightarrow}\right.
$$

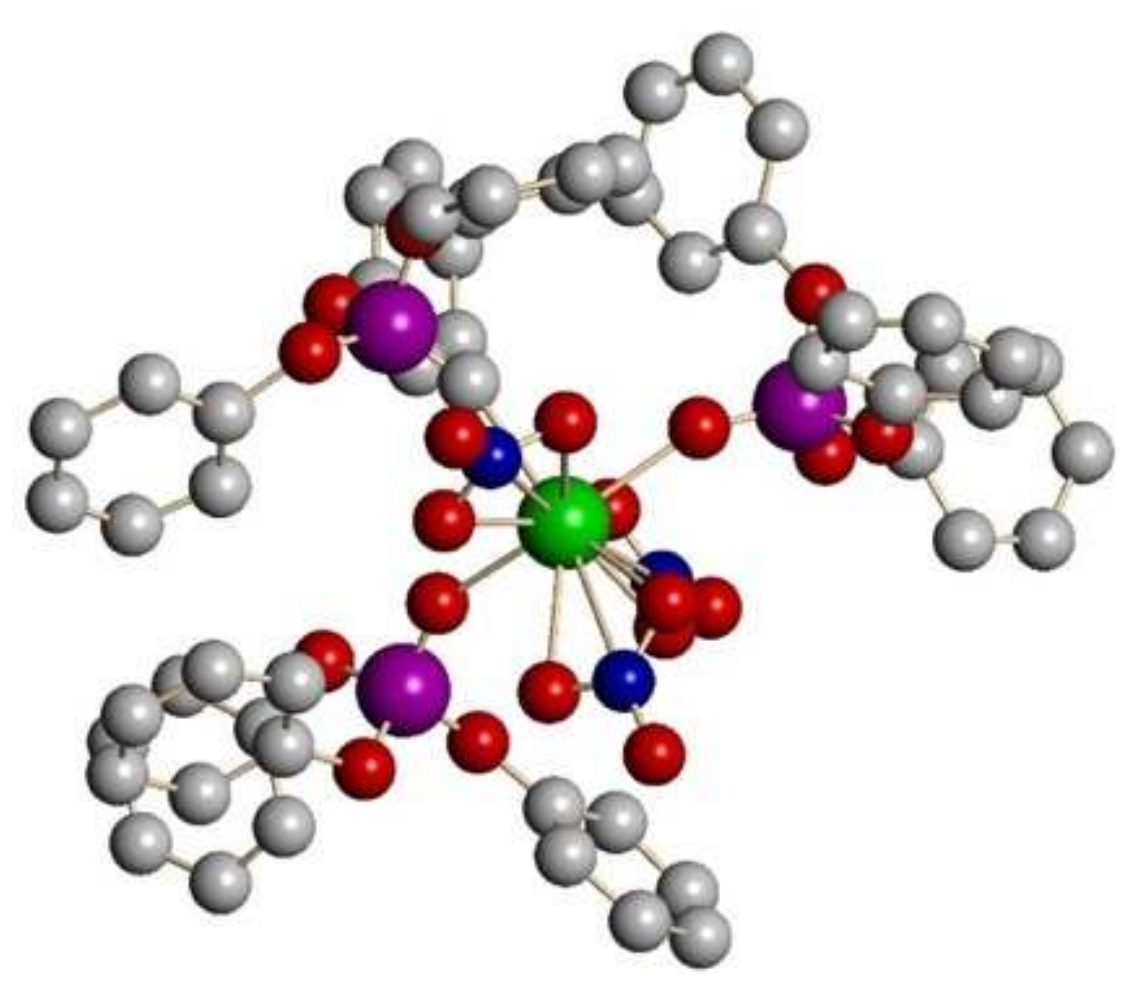

$\left[\mathrm{Ln}\left(\mathrm{NO}_{3}\right)_{3}(\mathrm{TPhP})_{3}\right]$

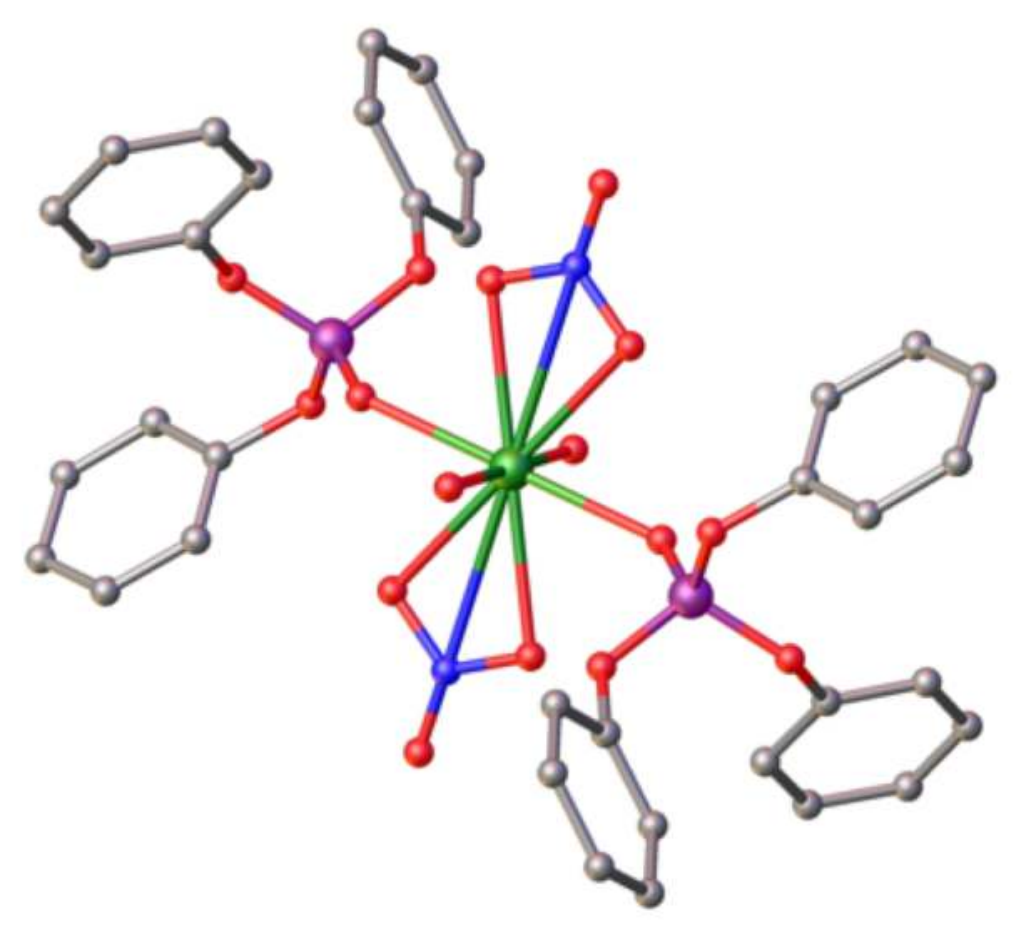

$\left[\mathrm{U}(\mathrm{O})_{2}\left(\mathrm{NO}_{3}\right)_{2}(\mathrm{TPhP})_{2}\right]$ 


\section{With solutions in hand, beaker heating of surrogates}

\section{were undertaken}

\section{- PUREX}

- Solvent-solvent extraction

- $30 \%$ TBP in Kerosene

- TBP molecule binds to $U$ and Pu
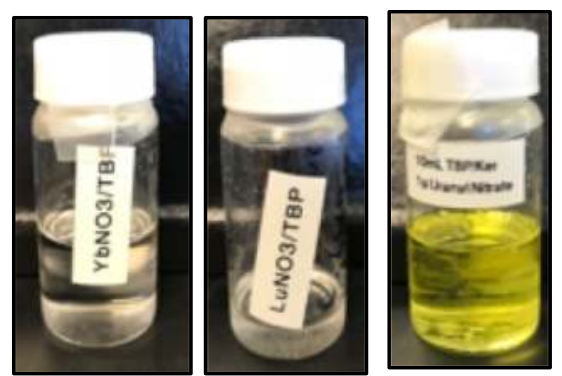

\begin{tabular}{lllll} 
Metal & $\begin{array}{l}\text { Assumed } \\
\text { Oxide }\end{array}$ & $\begin{array}{l}\text { Density } \\
\left(\mathrm{g} / \mathrm{cm}^{3}\right)\end{array}$ & $\begin{array}{l}\text { Solution } \\
(\mathrm{g} / \mathrm{mL})\end{array}$ & $\begin{array}{l}\text { Notes } \\
\mathrm{Ce}\end{array}$ \\
$\mathrm{CeO}_{2}$ & 7.2 & $\mathrm{~N} / \mathrm{A}$ & $\begin{array}{l}\text { Common surrogate, } \\
\text { not tested here }\end{array}$ \\
$\mathrm{Ho}$ & $\mathrm{Ho}_{2} \mathrm{O}_{3}$ & 8.1 & $\mathrm{~N} / \mathrm{A}$ & $\begin{array}{l}\text { Insoluble } \\
\mathrm{Sm}\end{array}$ \\
$\mathrm{Sm}_{2} \mathrm{O}_{3}$ & 8.3 & 0.1 & \\
$\mathrm{Er}$ & $\mathrm{Er}_{2} \mathrm{O}_{3}$ & 8.6 & 0.1 & \\
\hline $\mathrm{Yb}$ & $\mathrm{Yb}_{2} \mathrm{O}_{3}$ & 9.2 & 0.1 & Better surrogate \\
Lu & $\mathrm{Lu}_{2} \mathrm{O}_{3}$ & 9.4 & 0.1 & Better surrogate \\
\hline $\mathrm{Th}$ & $\mathrm{ThO}_{2}$ & 10.0 & - & Not yet tested \\
$\mathrm{U}$ & $\mathrm{UO}_{2}$ & 11.0 & 0.1 & $99.7 \%$ 238-U \\
\hline
\end{tabular}

\section{-What metals?}

- Soluble in TBP/Kerosene to similar level as U ( 120 g/L)

- High particle density so transport is similar

- Lanthanides often used as alternatives due to their similar size, electronics, densities, and additional inherent properties
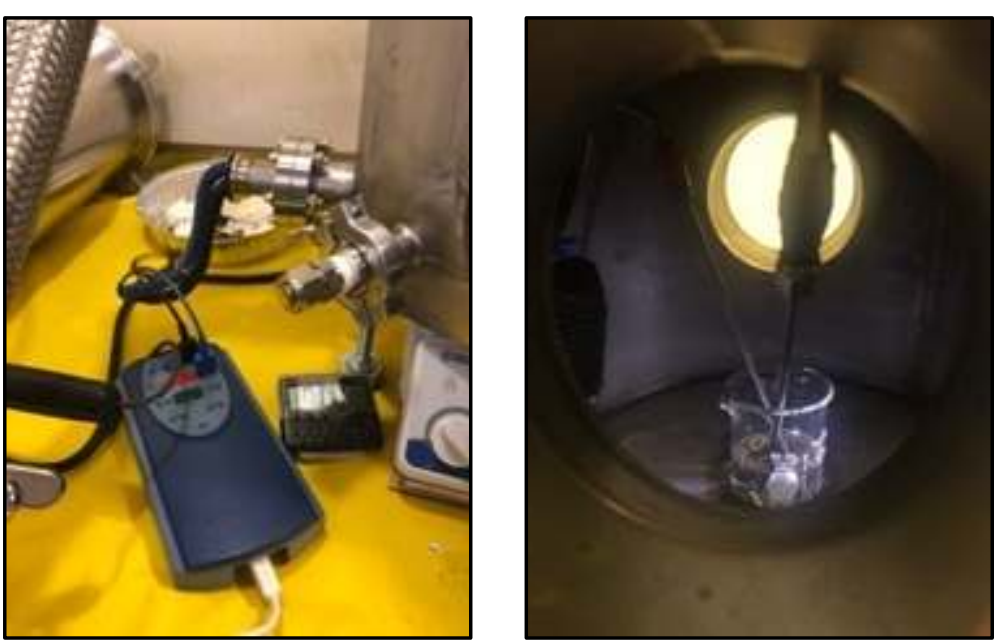

Non-combustion heating to determine rates and the probability that boiling conditions were attained during airborne release fraction (ARF) measurements.

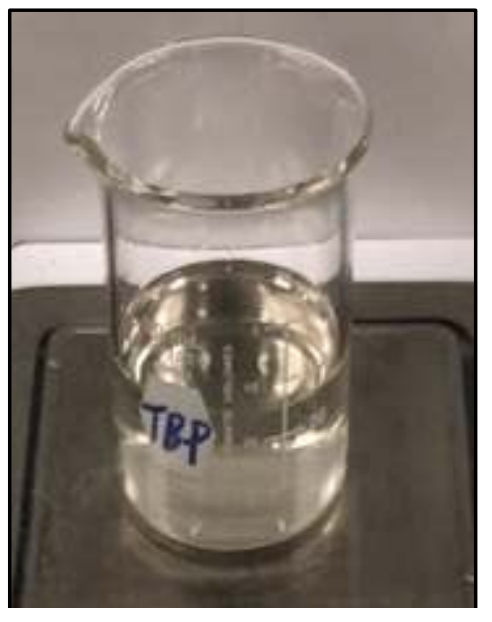

Heating up to $110^{\circ} \mathrm{C}$ when no metal-nitrates were present

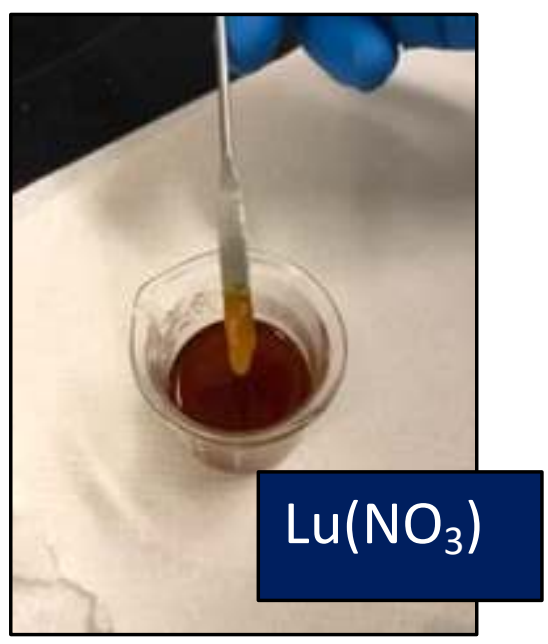

Heating up to $110^{\circ} \mathrm{C}$ caused phase separation 


\section{Test Chamber designed for optimal burning}

Distinguishing feature - porous walls and chamber bottom which bring in dilution air and prevents aerosol deposits.

\section{$\mathrm{CO}_{2}$ purge - fire extinguishing system}

Materials are completely enclosed - enables work with radioactive materials.

System sits inside a fume hood for secondary containment.

Metal, HEPA, and carbon filters downstream of aerosol capture filter visualization and instrumentation ports.

Aerosols are captured at the top of the chamber on EPM-2000 glass fiber filters, leached, and quantified with Inductively Coupled Plasma Mass Spectrometry (ICP-MS)
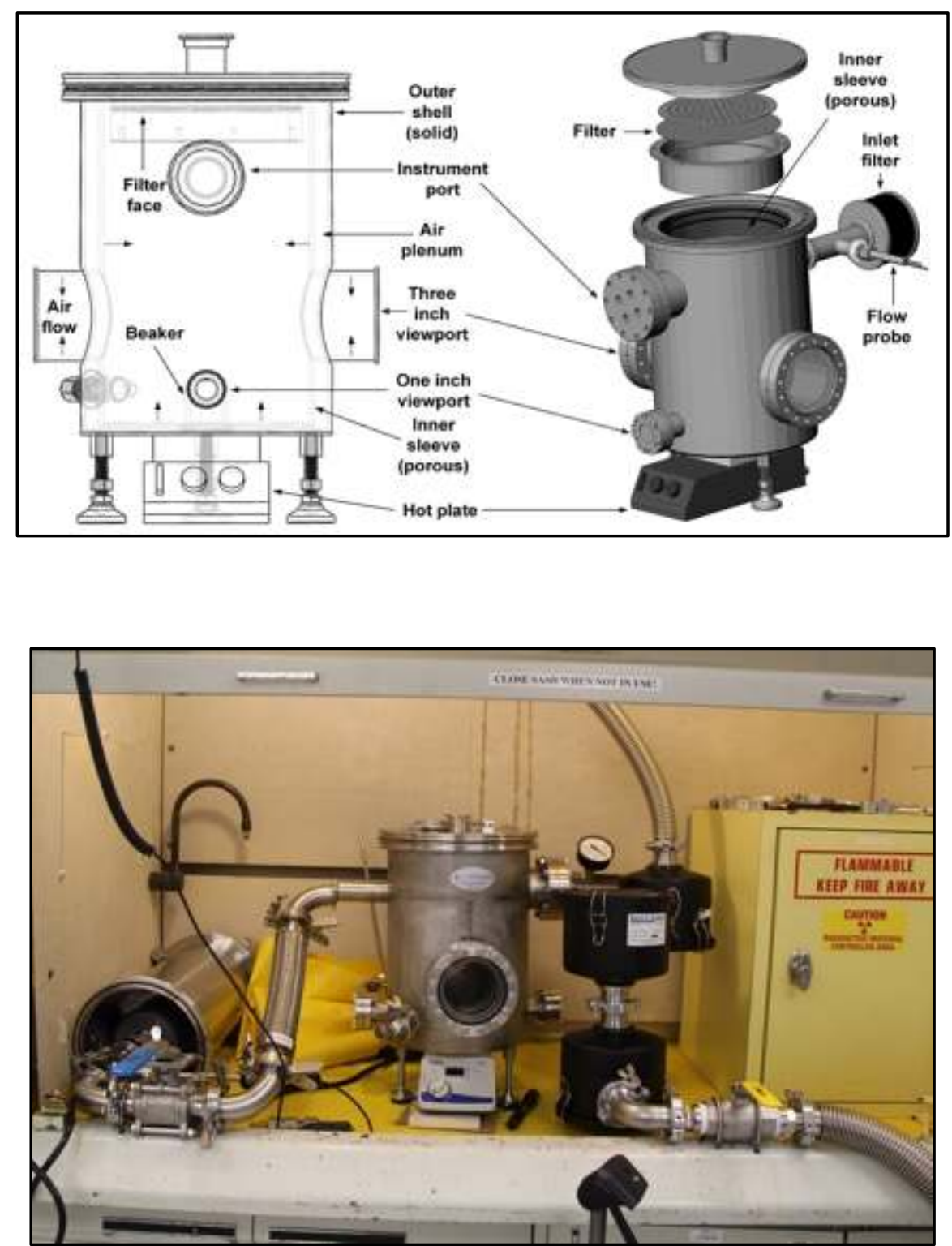


\section{Real time testing of burned samples of the Ln surrogate solutions}

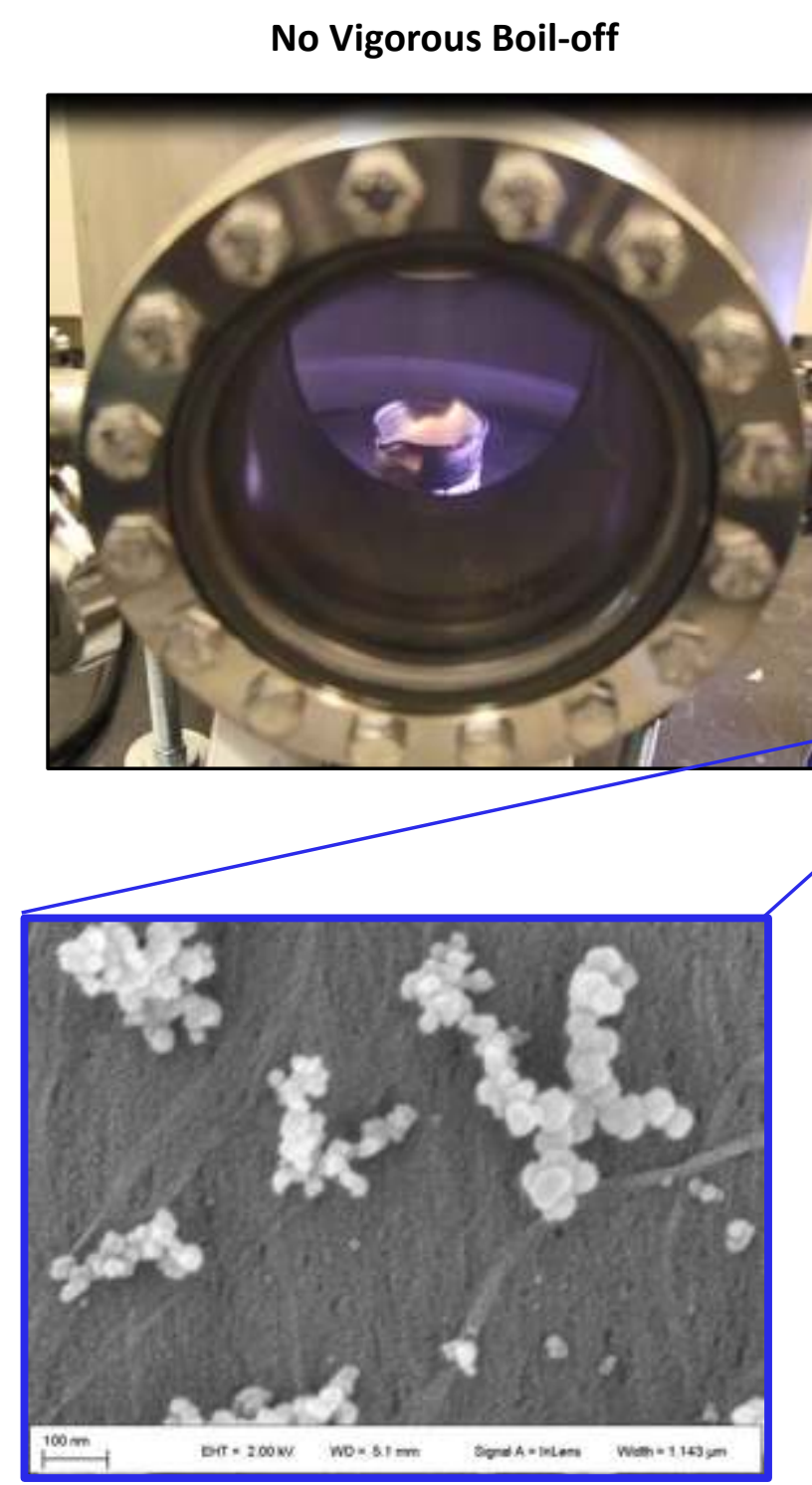

Fuel: $30 \%$ TBP in kerosene, no metal nitrate

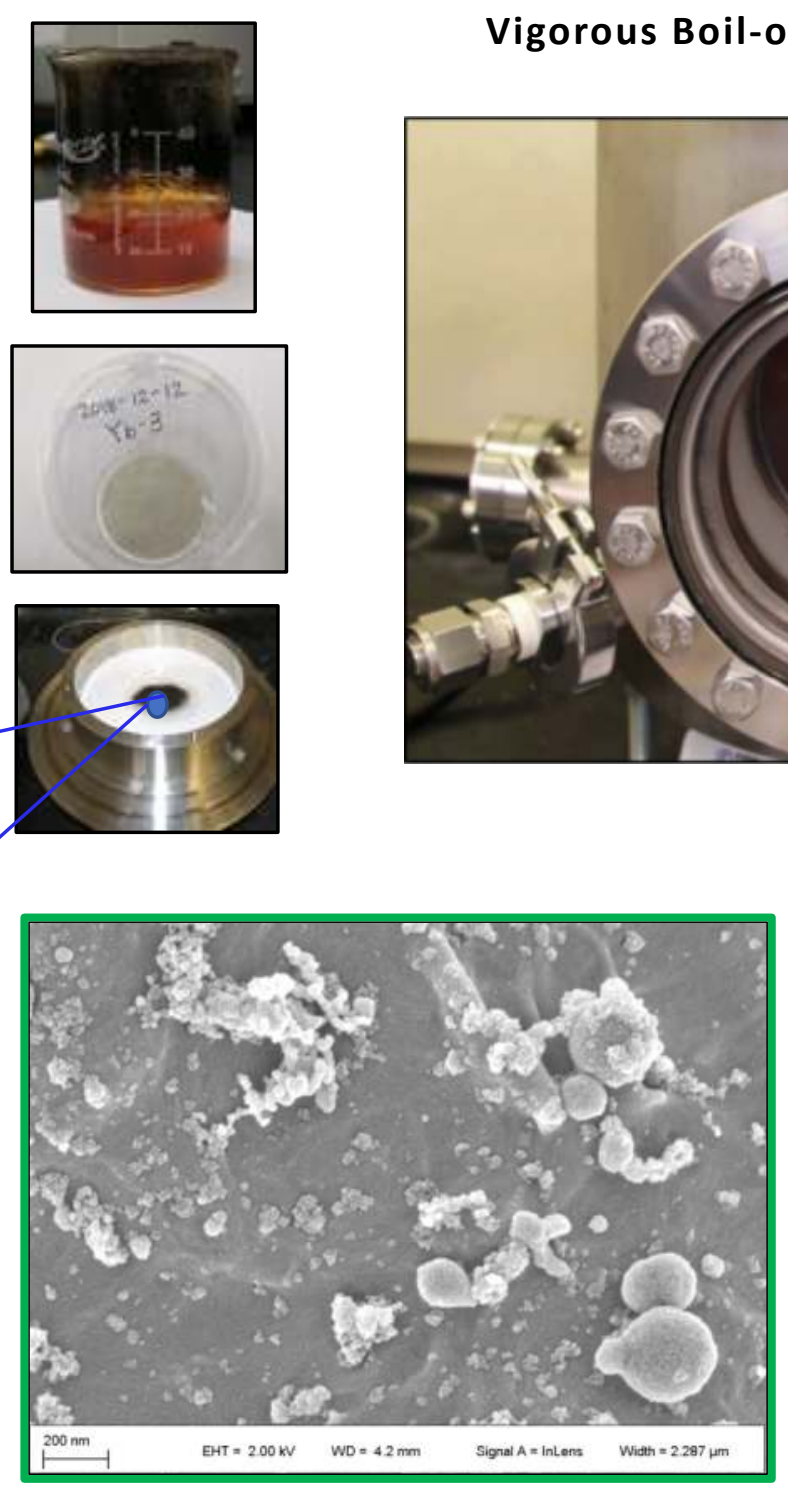

Fuel: 30\% TBP in kerosene, $0.1 \mathrm{~g} / \mathrm{mL}$ lutetium nitrate
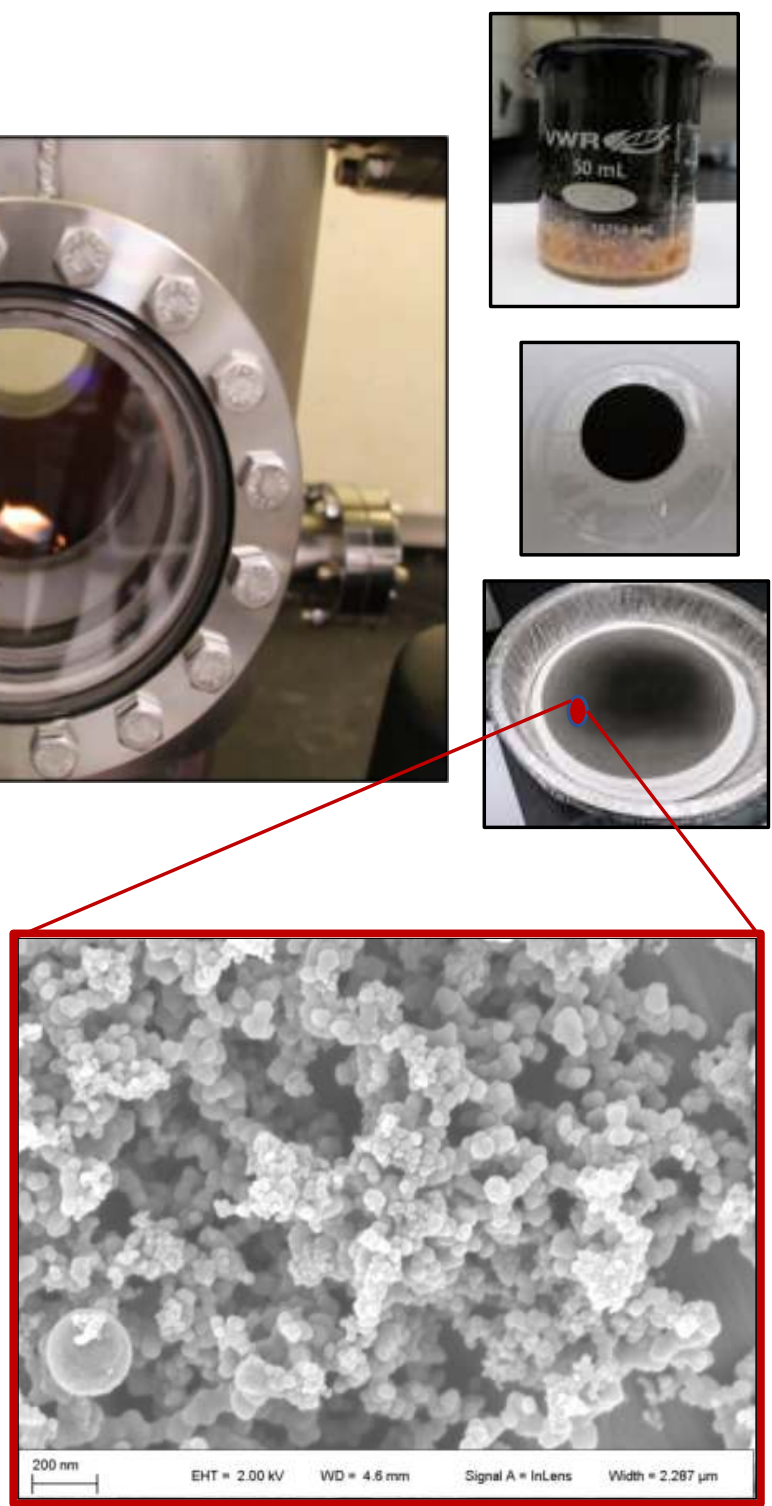

Fuel: $30 \%$ TBP in kerosene, $0.1 \mathrm{~g} / \mathrm{mL}$ ytterbium nitrate 
Filter Analysis used EPA standards to extract metal particulates (ARF) from filter paper
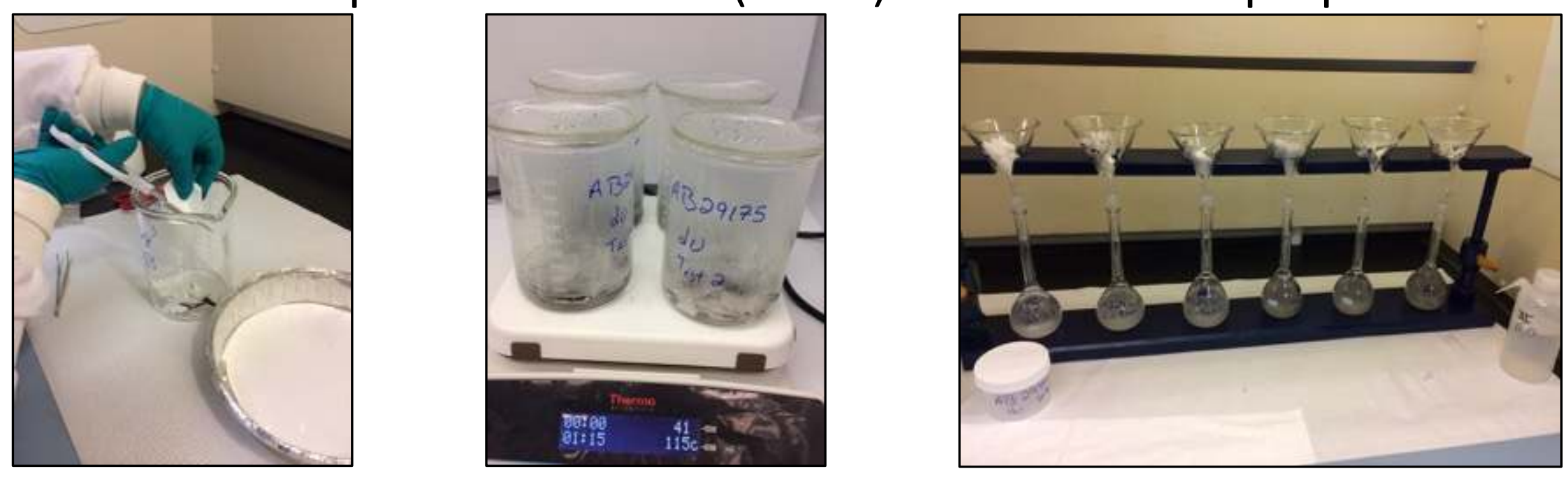

- 3 runs for each precursor (lutetium nitrate, ytterbium nitrate, and uranium nitrate)

- Filters collected for destructive analysis

- Leached metals from particulate, and performed Inductively Coupled Plasma Mass Spectrometry (ICP-MS) to quantify mass of metal on filters
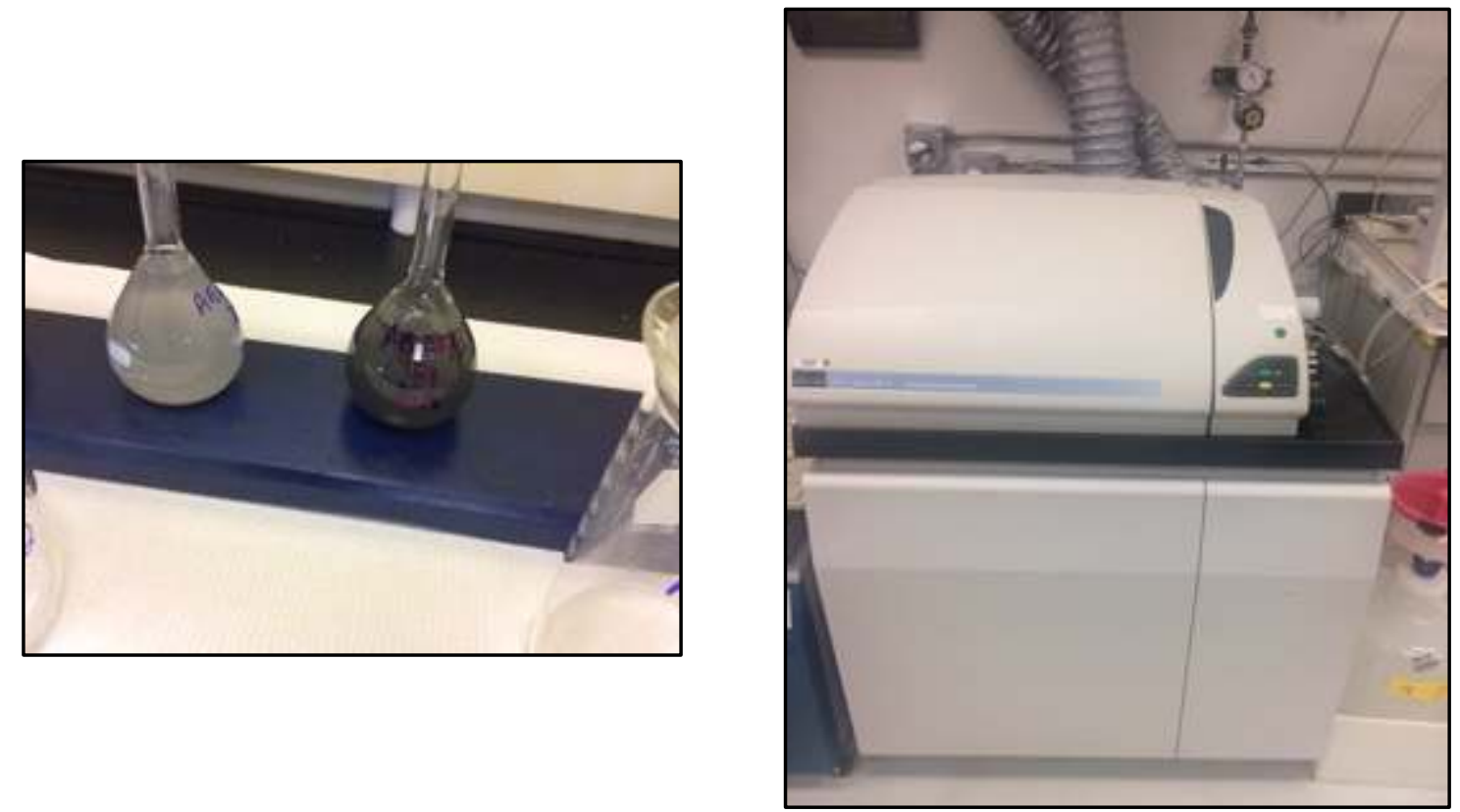


\section{Mass Loss and Time to Self Extinguishment}
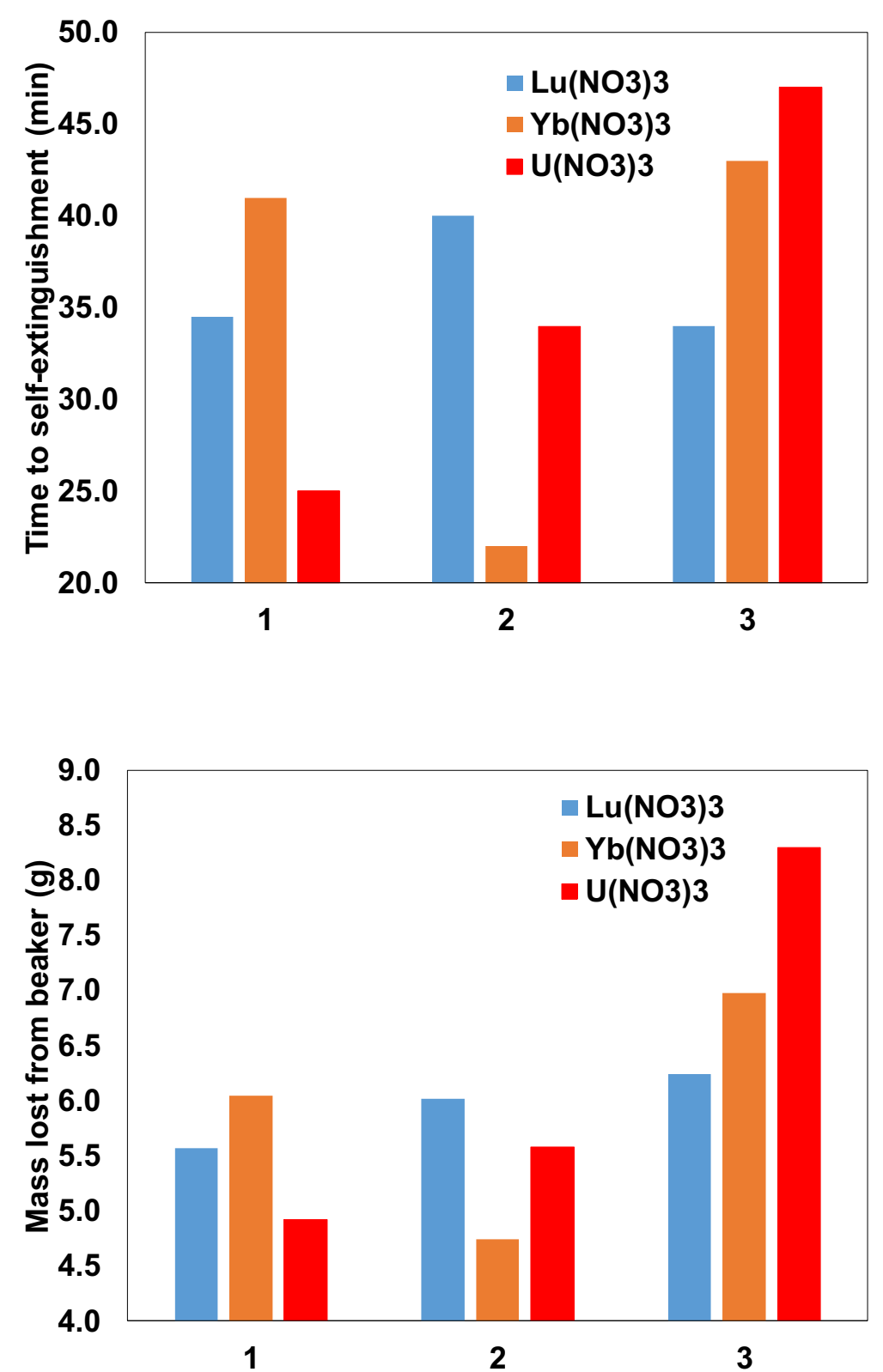

$25 \mathrm{~mL}$ sample took $\sim 40$ min to ignite with many additional periods of heating, flame appeared to undulate more than other experiments with $\mathrm{Yb}\left(\mathrm{NO}_{3}\right)_{3}$

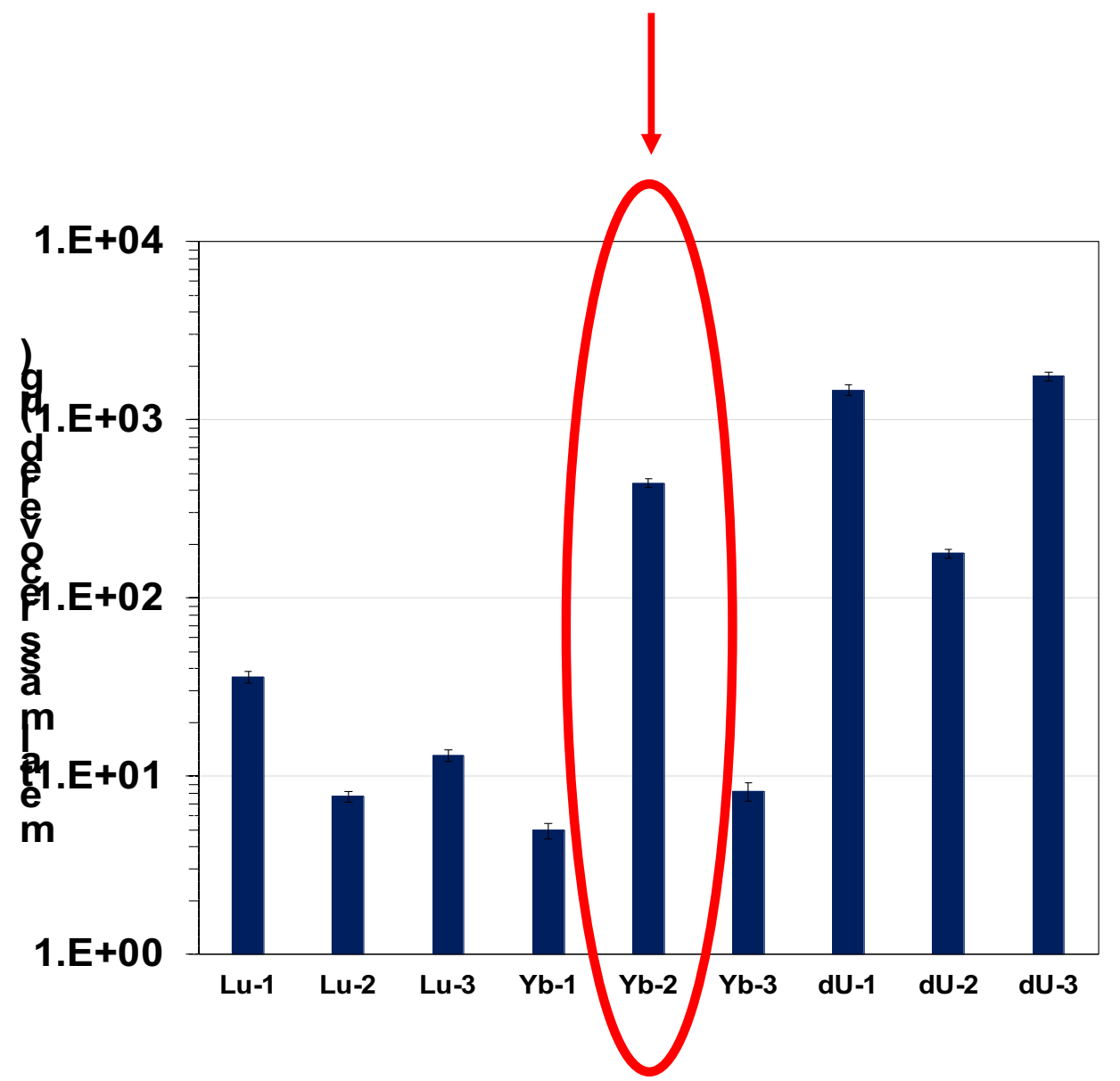

Analyzing ICP-MS data, uncertainties, and ARF 


\section{Lower ARF of $\mathrm{Yb}$ and Lu appear to be correlated to "additional heating intervals" - poor surrogates!}

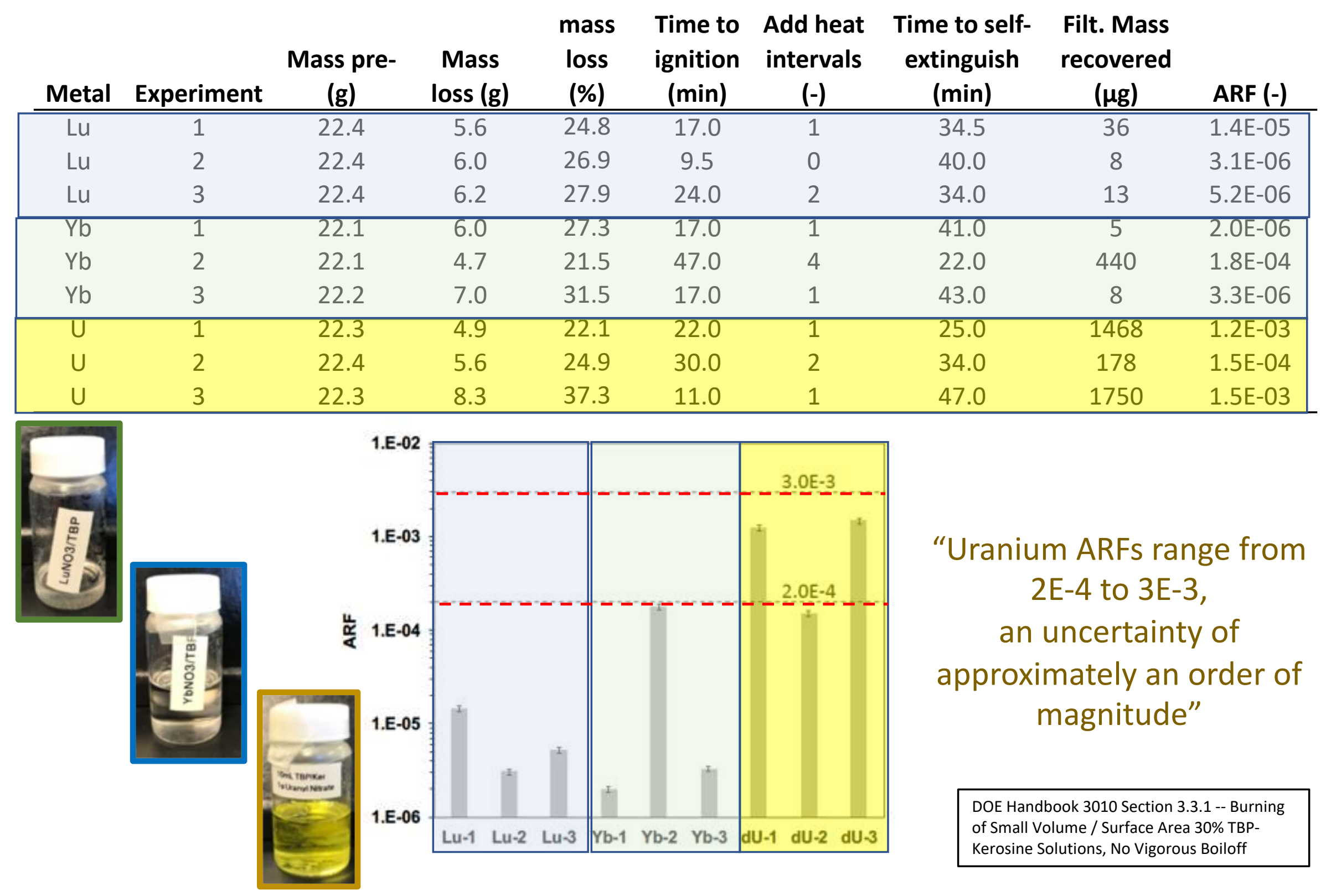


Processing of the $\mathrm{Ln}-\mathrm{NO}_{3}-\mathrm{O}=\mathrm{P}(\mathrm{OR})_{3}$ do not form the expected oxide but instead form the phosphate
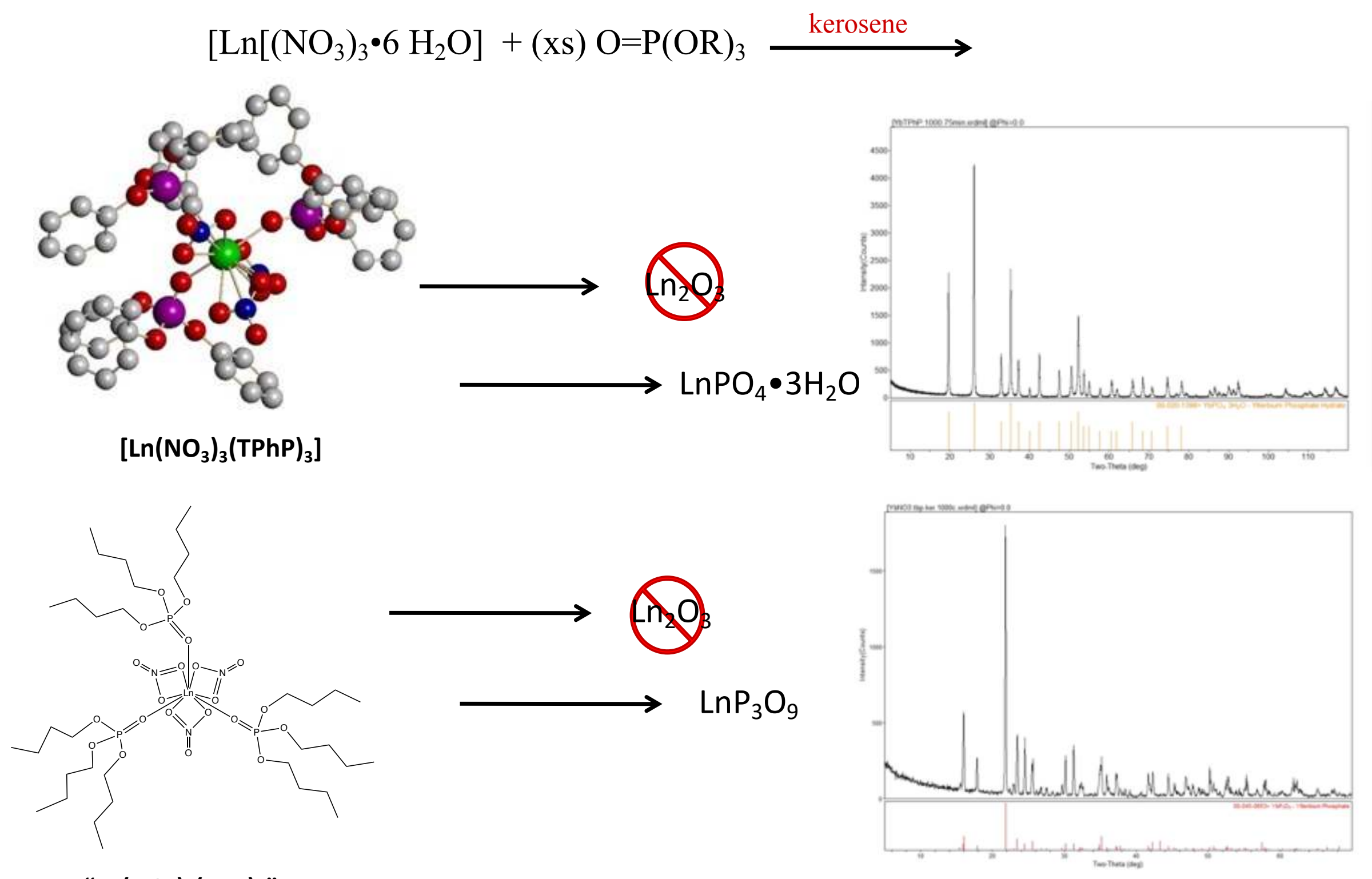

$" \operatorname{Ln}\left(\mathrm{NO}_{3}\right)_{3}(\mathrm{TBP})_{3} "$ 


\section{$\mathrm{U}(\mathrm{O})_{2}\left(\mathrm{NO}_{3}\right)_{2} \bullet 6 \mathrm{H}_{2} \mathrm{O}$ in the presence of $\mathrm{O}=\mathrm{P}(\mathrm{OR})_{3}$ also found to unexpectantly form the phosphate phase}

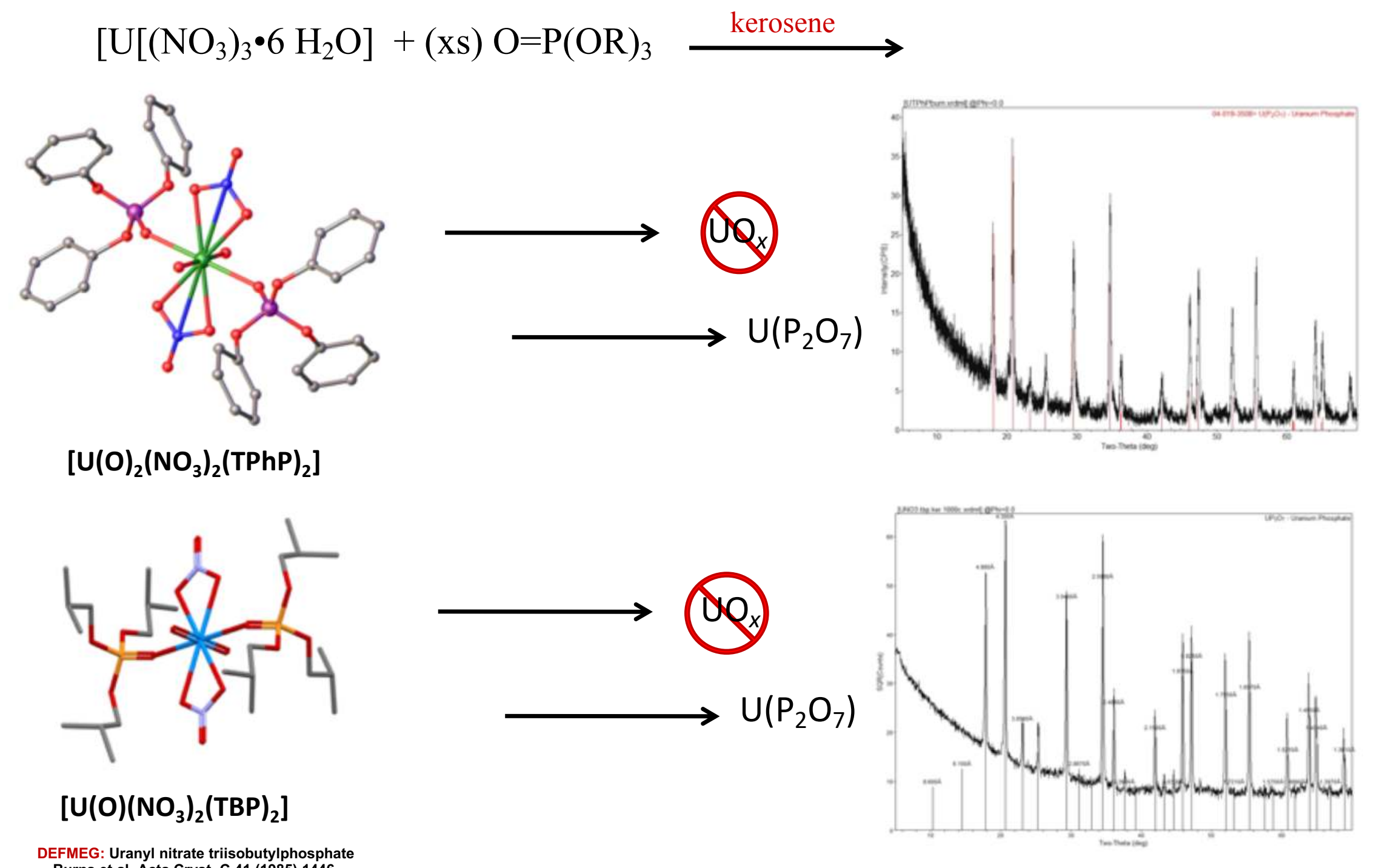




\section{Summary and Conclusions}

- Solubility of Ln show useful surrogate - heavier Ln of interest for aerosol studies.

- Coordination chemistry of $\left[\mathrm{Ln}\left(\mathrm{NO}_{3}\right)_{3}(\mathrm{TPhP})_{3}\right]$ determined (31P and SCXRD).

- H-DAA formed from $\left[\mathrm{Ln}\left(\mathrm{NO}_{3}\right)_{3}\left(\mathrm{H}_{2} \mathrm{O}\right)_{6}\right]$ in acetone.

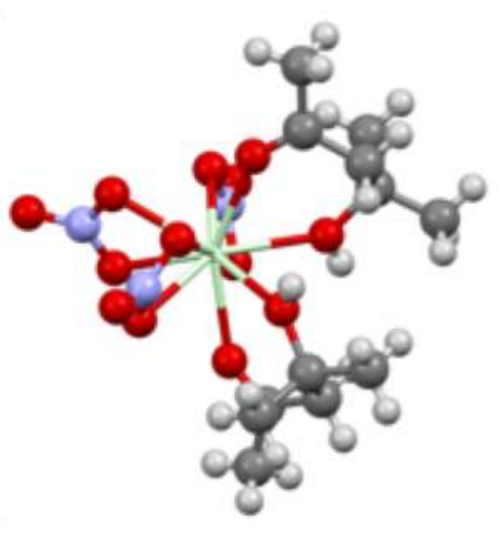

$\left[\operatorname{Ln}\left(\kappa^{2}-\mathrm{NO}_{3}\right)_{3}\left(\kappa^{2}-\mathrm{DAA}\right)_{2}\right]$

- Acid catalyzed room temperature conversion?

- Burning showed similar particulates and burn parameters for U- and Ln-system.

- Lower ARF for Ln-system: high temp solubility issues.

- Processing showed Ln-phosphate formed (but so did U-system)!

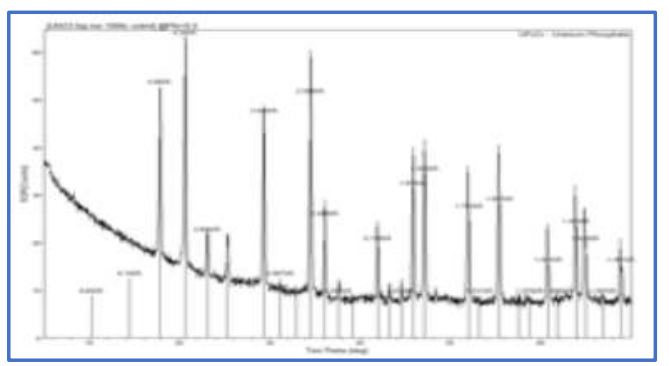

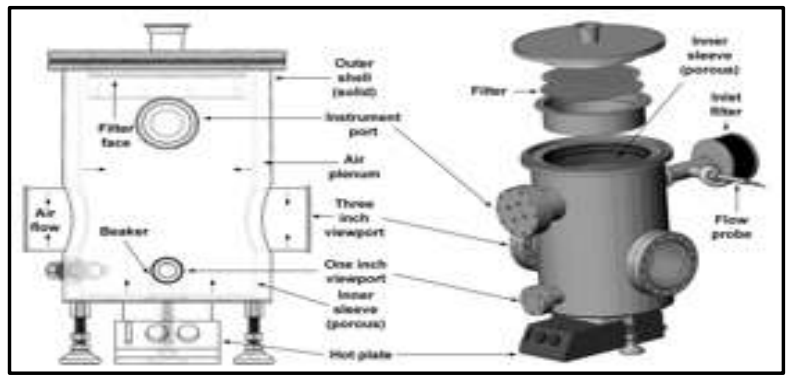

